\title{
Enlarging the space of viable inflation models: A slingshot mechanism
}

\author{
Keith R. Dienes, ${ }^{1,2 *}$ Jeff Kost $\odot,{ }^{3, \dagger}$ and Brooks Thomas $\oplus^{4, *}$ \\ ${ }^{1}$ Department of Physics, University of Arizona, Tucson, Arizona 85721 USA \\ ${ }^{2}$ Department of Physics, University of Maryland, College Park, Maryland 20742 USA \\ ${ }^{3}$ Center for Theoretical Physics of the Universe, Institute for Basic Science, Daejeon 34126 Korea \\ ${ }^{4}$ Department of Physics, Lafayette College, Easton, Pennsylvania 18042 USA
}

(Received 30 July 2019; published 11 October 2019)

\begin{abstract}
The viability of a given model for inflation is determined not only by the form of the inflaton potential, but also by the initial inflaton field configuration. In many models, field configurations which are otherwise well-motivated nevertheless fail to induce inflation, or fail to produce an inflationary epoch of duration sufficient to solve the horizon and flatness problems. In this paper, we propose a mechanism which enables inflation to occur even with such initial conditions. Our mechanism involves multiple scalar fields which experience a time-dependent mixing. This in turn leads to a "re-overdamping" phase as well as a parametric resonance which together "slingshot" the inflaton field from regions of parameter space that do not induce inflation to regions that do. Our mechanism is flexible, dynamical, and capable of yielding an inflationary epoch of sufficiently long duration. This slingshot mechanism can therefore be utilized in a variety of settings and thereby enlarge the space of potentially viable inflation models.
\end{abstract}

DOI: $10.1103 /$ PhysRevD.100.083516

\section{INTRODUCTION, MOTIVATION, AND SUMMARY}

Because of its many properties, both theoretical and observational, the inflationary paradigm has become a standard component of early-universe cosmology [1-6]. Indeed, many observations and cosmological fine-tuning problems-e.g., the horizon problem, the absence of primordial topological defects, the flatness problem-can be understood by positing an epoch of rapid inflationary expansion in the early universe. Moreover, models for inflation generally predict a nearly scale-invariant spectrum of primordial perturbations, in agreement with observations of the cosmic microwave background (CMB) and largescale structure [7-12].

One critical ingredient in any inflationary scenario is an appropriate scalar potential $V(\phi)$ for the inflaton $\phi$. The form of $V(\phi)$ is constrained by observations-in particular, inflation typically only occurs when $\phi$ traverses a sufficiently flat region of the potential. Likewise, an inflationary model also requires the specification of a set of initial conditions for the inflaton field within the phase space

\footnotetext{
*dienes@email.arizona.edu

†jeffkost@ibs.re.kr

thomasbd@lafayette.edu
}

Published by the American Physical Society under the terms of the Creative Commons Attribution 4.0 International license. Further distribution of this work must maintain attribution to the author(s) and the published article's title, journal citation, and DOI. Funded by SCOAP. $\{\phi, \dot{\phi}\}$. Only certain regions within this phase space give rise to inflationary dynamics, and only certain subregions thereof lead to an inflationary epoch of sufficient duration. Indeed, the number of $e$-folds required for successful inflation generally falls within a range $N_{\text {inf }} \gtrsim 50-60$, where the uncertainty is due to our ignorance of the amount of expansion which occurs during the reheating epoch [13]. As a result, the initial field configuration $\{\phi, \dot{\phi}\}$ is another critical ingredient in determining the phenomenological viability of a given inflation model.

As an example, let us consider perhaps the simplest inflaton potential, $V(\phi)=\frac{1}{2} m_{\phi}^{2} \phi^{2}$, where $m_{\phi}$ is the inflaton mass. In Fig. 1 we show the corresponding phase space $\{\phi, \dot{\phi}\}$, with contour lines indicating the number of $e$-folds of inflation that are eventually produced if our field begins at that location. We have also colored the different regions of this phase space accordingly, so that initial configurations within the red region do not lead to inflation at all while points within the gray region lead to insufficient inflation (defined for this figure as having fewer than 60 $e$-folds). Only the regions in blue lead to an inflationary epoch of sufficient duration.

These results can be understood in terms of the dynamical flow of the inflaton field within this phase space. In general, regardless of its initial location, the field will travel along an $e$-fold contour line in the direction of one of the black-dashed attractor curves at $\dot{\phi}= \pm \sqrt{2 / 3} m_{\phi} M_{p}$ where $M_{p}$ is the reduced Planck mass. Two sample flows are indicated in yellow in Fig. 1. Only when the inflaton 


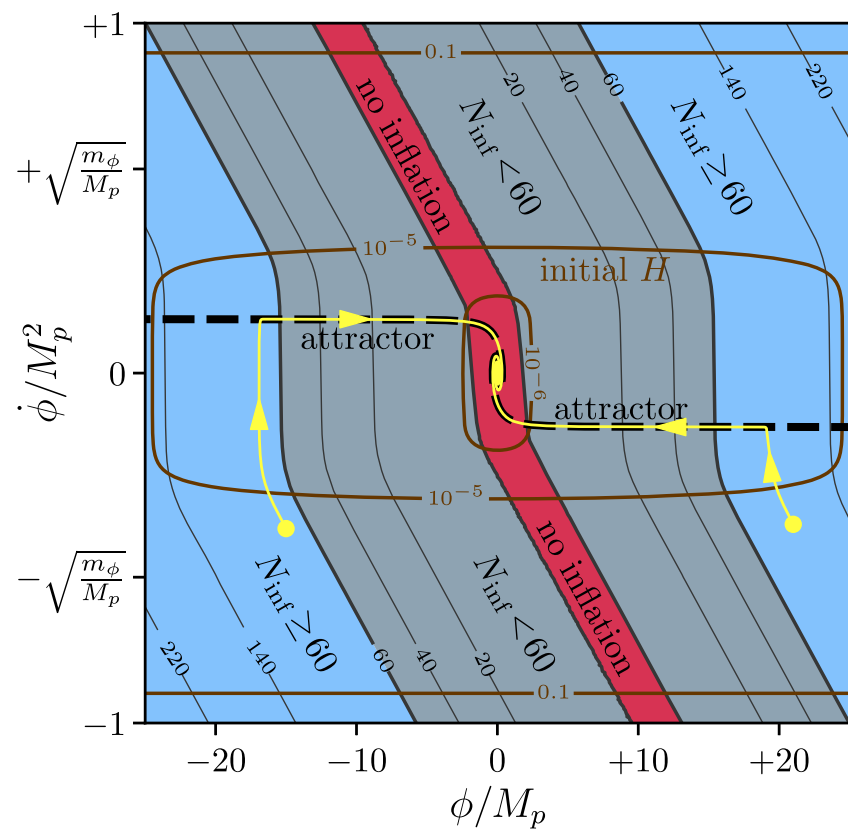

FIG. 1. The space of possible initial configurations $\{\phi, \dot{\phi}\}$ for the inflaton field in the case of a quadratic potential $V(\phi)=$ $\frac{1}{2} m_{\phi}^{2} \phi^{2}$ with $m_{\phi}=10^{-6} M_{p}$. The blue regions indicate the locations that can yield an inflationary epoch of duration [here taken to be $\gtrsim \mathcal{O}(60) e$-folds] sufficient to solve the horizon and flatness problems, while the gray regions lead to insufficient inflation and the red regions lead to no inflation at all. The brown contours indicate the initial Hubble scale $H_{\mathrm{I}}$ for each initial configuration $\{\phi, \dot{\phi}\}$, and two example trajectories (yellow) for the inflaton field are also shown. The black dashed curves show the attractors along which inflation occurs.

reaches the attractor does inflation begin, and only when the inflaton joins the attractor at sufficiently large $|\phi|$ will this inflationary epoch have sufficient duration. Thus, as a result of this dynamics, we see that the regions of phase space which lead to sufficiently long inflationary epochs are those with sufficiently large initial values of $|\phi|$ or $|\dot{\phi}|$ or both. Similar conclusions hold for a wide variety of potentials.

Within the context of such potentials, many different scenarios exist for generating such initial conditions involving large $|\phi|$ or $|\dot{\phi}|$. For example, in chaotic inflation [14], initial conditions are assumed to be randomly distributed, subject to a Planckian bound on the total energy density $\rho \leq M_{p}^{4}$. One then finds that for small $m_{\phi}$, the initial vacuum expectation values (VEVs) for $|\phi|$ and/or $|\dot{\phi}|$ are typically large, the former even trans-Planckian. Likewise, natural inflation $[15,16]$ also implements such a large field VEV for $|\phi|$, even if $|\dot{\phi}|$ in such scenarios is typically small.

Our focus here, by contrast, is on the opposite situation: what happens if both $|\phi|$ and $|\dot{\phi}|$ are initially small, perhaps both even sub-Planckian? After all, sub-Planckian field VEVs might be viewed as more natural from an effective field theory point of view. However, in many inflationary models, such initial field VEVs correspond to regions of phase space in which slow-roll conditions will be severely violated and inflation will not occur. Is there any way inflation can be salvaged in such scenarios?

In this paper, we shall propose a mechanism which accomplishes precisely this. We shall refer to this as a "slingshot" mechanism: even if we begin within a region of phase space from which inflation would not ordinarily be expected to occur, the dynamics of the system can "slingshot" the inflaton field into a different region of phase space in which it does. Our slingshot mechanism is built upon a brief time-dependent modification to the scalar potential in the inflationary sector of the theory-precisely as might arise as the consequence of a pre-inflationary cosmological phase transition. As we shall discuss, the resulting inflaton dynamics includes a short-lived but robust parametric resonance followed by a unique re-overdamped phase, and together these two features propel the inflaton field into new regions of phase space which potentially exhibit the desired large field VEVs. Indeed, we shall find that both of these features generically arise together in such scenarios. Our slingshot mechanism is therefore completely flexible, dynamical, and capable of giving rise to an inflationary epoch of sufficiently long duration. We also expect that our mechanism is also rather general, and can be utilized in a variety of different settings and for a variety of different potentials. This mechanism can thereby enlarge the space of potentially viable inflation models.

This paper is organized as follows. We begin in Sec. II by presenting the physics of our slingshot mechanism. As we shall discuss, the natural arena for this physics is a sector of scalar fields experiencing a mass-generating phase transition. Specifically, we shall focus our study on the simple yet illustrative example of a sector consisting of two real scalar fields. Then, in Sec. III, we embed this sector within the context of a general cosmology and demonstrate that our slingshot mechanism can indeed give rise to inflationeven when our initial field configuration is within otherwise "forbidden" regions such as the red region in Fig. 1. We investigate the general parameter space associated with our mechanism and determine those regions in which sufficient inflation can be produced. In this way we demonstrate that our mechanism can give rise to not only an inflationary epoch but also one with a number of $e$-folds sufficient for phenomenological purposes. In Sec. IV, we then develop an analytic approach to understanding the numerical results presented in Sec. III - an approach which allows us to forge a direct connection between the underlying parameters which characterize the phase transition and the relevant inflationary parameters and observables. In Sec. V we then exploit this connection in order to establish constraints on this minimal realization of our "slingshot" mechanism. Finally, in Sec. VI, we summarize our main results, elaborate on the various implications of our slingshot 
mechanism, and discuss a number of potential extensions and generalizations of the minimal scenario we have presented here. In Appendix A, we discuss constraints that can arise from particle production due to nonadiabatic changes in the vacuum state.

\section{THE SCALAR SECTOR AND THE SLINGSHOT MECHANISM}

As discussed in Sec. I, our slingshot mechanism is built upon the nontrivial dynamics of a two-scalar system which undergoes a mass-generating phase transition. In this section we shall therefore introduce our scalar system and discuss its dynamics, with the goal of understanding how and why a slingshot emerges.

\section{A. Two scalars and a mass-generating phase transition}

We begin by assuming the existence of a nonminimal scalar sector which experiences a mass-generating phase transition in the early universe, prior to an inflationary epoch. In particular, for concreteness we shall imagine that this sector consists of two scalar fields $\left\{\phi_{0}, \phi_{1}\right\}$ whose dynamics is governed by an effective potential $V_{\text {eff }}\left(\phi_{0}, \phi_{1}, t\right)$ which depends not only on the field VEVs $\phi_{0}$ and $\phi_{1}$ (thereby implicitly introducing a time dependence for $V_{\text {eff }}$ as our two fields evolve in field space) but also on other parameters which may carry their own explicit time dependence as the result of a possible phase transition. In a spatially flat Friedmann-Robertson-Walker (FRW) cosmology, the corresponding action for these fields then takes the form

$\mathcal{S}_{\phi}=\int d^{4} x \sqrt{-g}\left[\frac{1}{2}\left(\partial_{\mu} \phi_{0}\right)^{2}+\frac{1}{2}\left(\partial_{\mu} \phi_{1}\right)^{2}-V_{\text {eff }}\left(\phi_{0}, \phi_{1}, t\right)\right]$

where $g$ is the determinant of the FRW metric. While many algebraic forms for $V_{\text {eff }}\left(\phi_{i}, t\right)$ are possible, for simplicity we shall henceforth assume that the contributions to $V_{\text {eff }}\left(\phi_{i}, t\right)$ are of the form

$$
V_{\mathrm{eff}}\left(\phi_{i}, t\right)=\frac{1}{2} \sum_{i, j} \phi_{i} \mathcal{M}_{i j}^{2}(t) \phi_{j}
$$

where $\mathcal{M}^{2}(t)$ is the corresponding $2 \times 2$ time-dependent squared-mass matrix for our two scalars. Under this assumption, our cosmological phase transition can be viewed as a mass-generating phase transition. As we shall see, a sector consisting of only two scalar fields is sufficiently complex to give rise to all of the phenomena which shall eventually interest us, but also sufficiently simple that our analysis remains relatively straightforward and tractable. We emphasize, however, that there will be nothing in our eventual results that requires that there be only two fields in this scalar sector. Indeed similar phenomena can emerge in more complicated scenarios.

In order to maintain as much generality as possible, we shall follow Refs. $[17,18]$ in parametrizing the effects of our phase transition on the mass matrix $\mathcal{M}^{2}$. First, we shall let $t_{G}$ denote the time at which the phase transition occurs and assume that at early times $t \ll t_{G}$ the mass matrix takes the simple form

$$
\mathcal{M}^{2}=\left[\begin{array}{cc}
0 & 0 \\
0 & M^{2}
\end{array}\right] \quad \text { for } t \ll t_{G} .
$$

In other words, we are assuming that our lighter field is initially massless while our heavier field has a nonzero mass $M$. Next, we shall assume that our phase transition produces additional contributions to this mass matrix such that for times $t \gg t_{G}$ long after the phase transition our mass matrix takes the late-time asymptotic form

$$
\mathcal{M}^{2}=\left[\begin{array}{cc}
0 & 0 \\
0 & M^{2}
\end{array}\right]+\left[\begin{array}{cc}
\bar{m}_{00}^{2} & \bar{m}_{01}^{2} \\
\bar{m}_{01}^{2} & \bar{m}_{11}^{2}
\end{array}\right] \quad \text { for } t \gg t_{G},
$$

where $\bar{m}_{i j}^{2}$ represent the late-time contributions to the mass matrix arising from the phase transition. In this way, we allow for the possibility that our phase transition not only modifies the masses of our states, but also introduces a nontrivial mixing between them. Finally, we shall make the natural assumption that the phase transition unfolds smoothly in such a way that these extra contributions to $\mathcal{M}^{2}$ are all generated with a uniform time dependence. In other words, for any arbitrary time $t$, we shall assume that the mass matrix takes the form

$$
\mathcal{M}^{2}(t)=\left[\begin{array}{cc}
0 & 0 \\
0 & M^{2}
\end{array}\right]+\left[\begin{array}{cc}
m_{00}^{2}(t) & m_{01}^{2}(t) \\
m_{01}^{2}(t) & m_{11}^{2}(t)
\end{array}\right],
$$

where we can write

$$
m_{i j}(t)=\bar{m}_{i j} \cdot h(t)
$$

with $h(t)$ representing a monotonic function $h(t)$ such that $h(t) \rightarrow 0$ as $t / t_{G} \rightarrow 0$ and $h(t) \rightarrow 1$ as $t / t_{G} \rightarrow \infty$. This function $h(t)$ completely characterizes the manner in which the phase transition unfolds as a function of $t$. We shall enforce our expectation that $t_{G}$ is the "central time" of the phase transition by defining $t_{G}$ through the relation $h\left(t_{G}\right)=1 / 2$. Furthermore, the most significant changes to $h(t)$ occur during a window of approximate duration $\Delta_{G}$ centered around $t_{G}$-i.e., within the time interval $\left[t_{G}-\Delta_{G} / 2, t_{G}+\Delta_{G} / 2\right]$. Note that the mass matrix $\mathcal{M}^{2}$ in Eq. (2.5) is Hermitian and has non-negative eigenvalues at any instant in time so long as all of the $m_{i j}^{2}$ are real, with $m_{00}^{2} \geq 0, m_{11}^{2} \geq-M^{2}$, and $m_{00}^{2}\left(M^{2}+m_{11}^{2}\right) \geq m_{01}^{4}$. 
Beyond these requirements, most of the qualitative results of this paper are largely independent of the particular choice of functional form for $h(t)$. Indeed, for many purposes we may regard $h(t)$ rather than $t$ itself as our cosmological clock variable, choosing to study the dynamics of our system relative to our " $h$-clock" without worrying about the particular mapping between $h(t)$ and the true cosmological time $t$. However, when necessary, we shall adopt the specific choice $[17,18]$

$$
h(t)=\frac{1}{2}\left\{1+\operatorname{erf}\left[\frac{\sqrt{\pi} t_{G}}{\Delta_{G}} \log \left(\frac{t}{t_{G}}\right)\right]\right\},
$$

where the parameter $\Delta_{G}$, as discussed above, represents the approximate time interval over which the phase transition unfolds. Indeed, as discussed in Ref. [17], the algebraic form in Eq. (2.7) satisfies all of our requirements and provides a compelling model for a generic phase transition, provided that we take $\Delta_{G} \leq \sqrt{2 \pi} t_{G}$.

Given the action in Eq. (2.1), our fields $\phi_{0,1}$ then evolve according to the equations of motion

$$
\ddot{\phi}_{i}+3 H \dot{\phi}_{i}+\sum_{j} \mathcal{M}_{i j}^{2} \phi_{j}=0,
$$

where $H(t)$ is the Hubble parameter. With the mass matrix given in Eq. (2.5), the dynamics of the scalar sector is thus nearly completely determined. Indeed, it remains only to specify initial conditions for our fields at some initial time $t_{\star}$ prior to the phase transition.

Given our ultimate goal of extending the space of initial field configurations for inflation into regions that would a priori experience no inflation at all, a particularly convenient choice of initial conditions is to assume that only our initially massless field $\phi_{0}$ has a nonzero displacement, i.e., that $\phi_{i}\left(t_{\star}\right)=\mathcal{A}_{\phi} \delta_{i 0}$ where $\mathcal{A}_{\phi}$ is a constant, and that both fields start from rest, with $\dot{\phi}_{i}\left(t_{\star}\right)=0$. This choice of initial conditions ensures that our scalar sector has no energy prior the phase transition, so that all energy in the scalar sector is derived from the phase transition itself. Note that it is natural to take $\phi_{1}\left(t_{\star}\right)=\dot{\phi}_{1}\left(t_{\star}\right)=0$ because the heavy mass $M$ of this field renders it initially underdamped, whereupon Hubble friction damps out any oscillations this field might undergo. We can therefore assume that this is what has occurred prior to $t_{\star}$. However, there are also several reasons why we choose $\dot{\phi}_{0}\left(t_{\star}\right)=0$. First, even for a massless (and therefore overdamped) field $\phi_{0}$, Hubble friction damps out any initial field velocity $\dot{\phi}_{0}$ over a Hubble timescale, even while $\phi_{0}$ remains nonzero. Moreover, we note that an initial condition with nonzero $\phi_{0}$ but zero $\dot{\phi}_{0}$ emerges naturally from production mechanisms such as misalignment production. But most importantly, as explained in Sec. I, our goal in this paper is to have our initial values of $\phi_{i}$ and $\dot{\phi}_{i}$ both sub-Planckian.
While our eventual results will prove somewhat sensitive to $\mathcal{A}_{\phi}$ (which is why we shall keep this as a free parameter throughout this paper), most of our results will turn out to be largely insensitive to the precise sub-Planckian value of $\dot{\phi}_{0}\left(t_{\star}\right)$ as long as this value is of the same sign as $\mathcal{A}_{\phi}$. For all of these reasons, we shall assume that $\dot{\phi}_{0}\left(t_{\star}\right)=0$ in what follows. However, in Sec. III we shall return to this issue and demonstrate explicitly how our results would change if we allowed $\dot{\phi}_{0}\left(t_{\star}\right)$ to vary.

Our choice of the initial time $t_{\star}$ also deserves comment. Clearly we wish to choose a time which is much earlier than our phase transition, while our $h(t)$ function in Eq. (2.7) is still fairly close to zero. We shall therefore define our fiducial time $t_{\star}$ through the condition that $h\left(t_{\star}\right)=10^{-10}$, and we shall use this value for $t_{\star}$ for all explicit calculations in this paper. In particular, this choice is always larger than the Planck time, and for values of $t_{G}$ and $\Delta_{G}$ within what will eventually be our main region of phenomenological interest we find $t_{\star} \approx t_{G}-2.3 \Delta_{G}$. However, the main qualitative results of this paper will be completely independent of $t_{\star}$ so long as $t_{\star} \ll t_{G}$.

This, then, completely specifies the physics of the scalar sector. In particular, for any choice of the initial conditions $M$ and $\mathcal{A}_{\phi}$, we see that there are five parameters which govern the resulting physics: two parameters $t_{G}$ and $\Delta_{G}$ which govern the temporal features of the phase transition, and three parameters $\bar{m}_{00}^{2}, \bar{m}_{11}^{2}$, and $\bar{m}_{01}^{2}$ which describe the late-time contributions to the mass matrix that result from this phase transition. Because of the time-dependent nature of the mass matrix, our two fields $\phi_{0}$ and $\phi_{1}$ experience a nontrivial time evolution. In particular, at any instant of time these two fields experience a mixing angle $\theta(t)$ given by

$$
\tan (2 \theta) \equiv \frac{2 m_{01}^{2}}{M^{2}-m_{00}^{2}+m_{11}^{2}}
$$

Thus, the mass eigenstates of our system $\phi_{\lambda_{0}}$ and $\phi_{\lambda_{1}}$ along with their corresponding mass eigenvalues $\lambda_{0}$ and $\lambda_{1}$-are continually changing over the course of the phase transition. At any instant, the mass eigenstate $\phi_{\lambda_{i}}(t)$ is overdamped if $\lambda_{i}(t)<3 H(t) / 2$ and otherwise underdamped. Indeed it is only at late times $t \gg t_{G}$ after the phase transition has passed that our system asymptotes to one with particular fixed late-time mass eigenstates $\phi_{\lambda_{0}}$ and $\phi_{\lambda_{1}}$ with fixed late-time masses $\bar{\lambda}_{0}$ and $\bar{\lambda}_{1}$.

For our work, it shall prove convenient to recast the mass matrix (2.5) into the form

$$
\mathcal{M}^{2}=\frac{1}{2} m_{\text {sum }}^{2} \sqrt{1-\alpha^{2}}\left[\begin{array}{cc}
\sqrt{\frac{1-\alpha}{1+\alpha}} & 1-\beta \\
1-\beta & \sqrt{\frac{1+\alpha}{1-\alpha}}
\end{array}\right],
$$


where we have defined the time-dependent quantities

$$
\begin{aligned}
m_{\text {sum }}^{2} & \equiv M^{2}+m_{00}^{2}+m_{11}^{2}, \\
\alpha & \equiv \frac{M^{2}-m_{00}^{2}+m_{11}^{2}}{M^{2}+m_{00}^{2}+m_{11}^{2}} \\
\beta & \equiv 1-\frac{m_{01}^{2}}{\sqrt{m_{00}^{2}\left(M^{2}+m_{11}^{2}\right)}} .
\end{aligned}
$$

Thus $\beta$-the only one of these quantities which depends on $m_{01}^{2}$-parametrizes the degree to which our fields mix at any instant of time. Requiring a Hermitian, positivesemidefinite mass matrix in Eq. (2.10) then restricts us to the parameter range $m_{\text {sum }}^{2} \geq 0,|\alpha| \leq 1$, and $0 \leq \beta \leq 2$, with $\alpha= \pm 1$ allowed only for $\beta=1$. Indeed, within these ranges, Eq. (2.9) now takes the form

$$
\tan (2 \theta)=\frac{1-\beta}{\alpha} \sqrt{1-\alpha^{2}}
$$

where $-\pi \leq \theta \leq \pi$. Likewise, at any moment in time, the eigenvalues of the mass matrix in Eq. (2.10) take the simple form

$$
\lambda_{0,1}^{2}=\frac{1}{2} m_{\text {sum }}^{2}\left[1 \mp \sqrt{\alpha^{2}+\left(1-\alpha^{2}\right)(1-\beta)^{2}}\right],
$$

with $\lambda_{0}^{2}+\lambda_{1}^{2}=m_{\text {sum }}^{2}$, as expected. Since $\lambda_{0} \leq \lambda_{1}$ in all cases, we see that $m_{\text {sum }}^{2} / 2$ is both the maximum possible value for $\lambda_{0}^{2}$ and the minimum possible value for $\lambda_{1}^{2}$.

It is possible to exploit certain symmetries of this twoscalar model to restrict the ranges of our parameters still further. For example, as long as we are only interested in understanding the behavior of our fields and their corresponding energy densities, our system is invariant under the transformation $\left(\phi_{0}, \phi_{1}\right) \rightarrow-\left(\phi_{0}, \phi_{1}\right)$ in which the signs of both fields are simultaneously flipped. This symmetry allows us to restrict our attention to the range $-\pi / 2 \leq$ $\theta \leq \pi / 2$. Likewise, flipping the relative signs of the fields -e.g., taking $\left(\phi_{0}, \phi_{1}\right) \rightarrow\left(\phi_{0},-\phi_{1}\right)$-is tantamount to flipping $\beta \rightarrow 2-\beta$. We can thus restrict our attention to values of $\beta$ which lie within the range $0 \leq \beta \leq 1$ without loss of generality. This corresponds to restricting $m_{01}^{2} \geq 0$, or equivalently restricting our attention to $-\pi / 4 \leq \theta \leq$ $\pi / 4$, with positive (negative) values of $\theta$ corresponding to positive (negative) values of $\alpha$.

Thus, for any values of $M$ and $\mathcal{A}_{\phi}$, our scalar sector can be parametrized in terms of $\bar{m}_{\text {sum }}^{2}, \bar{\alpha}, \bar{\beta}, t_{G}$, and $\Delta_{G}$. However, for any value of $\bar{m}_{\text {sum }}^{2}$ and $\bar{\alpha}$, our result in Eq. (2.13) provides a one-to-one relationship between $\bar{\beta}$ and the late-time mass $\bar{\lambda}_{0}$ of the lighter scalar $\phi_{\lambda_{0}}$. Since $\phi_{\lambda_{0}}$ will eventually be identified with the inflaton, and $\bar{\lambda}_{0}$ with the inflaton mass, we will occasionally trade $\bar{\beta}$ for $\bar{\lambda}_{0}$ in what follows.

\section{B. Building the slingshot: Assembling the required ingredients}

In general, this two-field scalar system can exhibit a variety of different behaviors and thereby give rise to a rich set of possible phenomenologies [17]. However, what shall concern us in this paper is the possibility of a "slingshot" effect in which the VEV of the lighter field $\phi_{\lambda_{0}}$ is propelled toward super-Planckian values. We shall therefore henceforth focus on this possibility, and demonstrate that the required ingredients emerge quite naturally within the setup we have described.

In order to understand how this dynamics emerges, let us begin by considering the behavior of the lighter mass eigenvalue $\lambda_{0}$ as a function of the three parameters $\left\{m_{\text {sum }}^{2}, \alpha, \beta\right\}$. The value of $\lambda_{0}$ is given in Eq. (2.13), with contours of constant $\lambda_{0}^{2}$ plotted in Fig. 2 as fractions of its maximum value $m_{\text {sum }}^{2} / 2$. For this figure we have taken $m_{\text {sum }}^{2}=M^{2}$ and plotted contours within the remaining $(\alpha, \beta)$ parameter space.

Superimposed on this contour plot we have also indicated various "flow" lines, denoted with arrows. These lines indicate the time evolution of the system, and may be understood as follows. Long before the phase transition, our mass matrix takes the form given in Eq. (2.3),

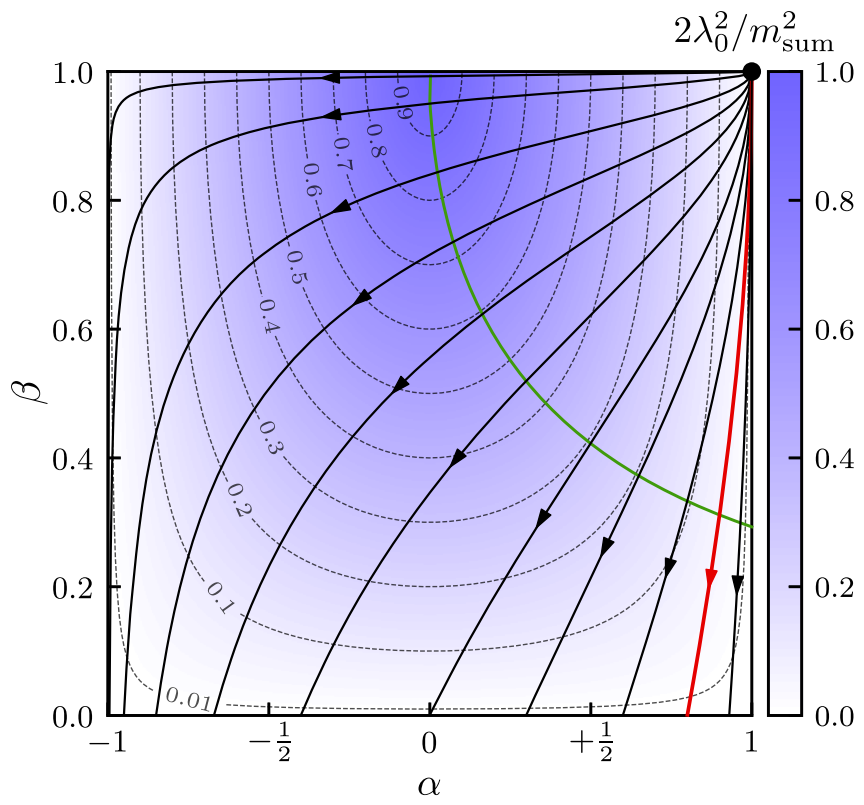

FIG. 2. A contour plot of the lighter eigenmass $\lambda_{0}^{2}$, normalized by its maximum possible value $m_{\text {sum }}^{2} / 2$, across the $\{\alpha, \beta\}$ parameter space. For this plot we have taken $m_{\text {sum }}^{2}=M^{2}$, with $M=M_{p} / 3$. Superimposed on this contour plot are "flow lines" (indicated with arrows), whose interpretations are discussed in the text. When needed for concreteness later in this paper, we shall often consider the "benchmark" flow line with $\bar{\alpha}=0.8$ and $\bar{\beta} \ll 1$ shown in red. An additional green contour line connects the points along each flow line at which the value of $\lambda_{0}$ is maximized. 
corresponding to $m_{\text {sum }}^{2}=M^{2}$ and $\alpha=\beta=1$. This is therefore the initial point for our time flow. In general, our system then evolves through different values $\left(m_{\text {sum }}^{2}\right.$, $\alpha, \beta)$ as functions of time, and the particular path that is followed through this parameter space ultimately depends on the values of the chosen late-time parameters $\bar{m}_{i j}^{2}$ [or equivalently $\left.\left(\bar{m}_{\text {sum }}^{2}, \bar{\alpha}, \bar{\beta}\right)\right]$ which parametrize the particular phase transition under study. However, for the special case $\bar{m}_{\text {sum }}^{2}=M^{2}$ (or equivalently $\bar{m}_{11}^{2}=-\bar{m}_{00}^{2}$ ), it turns out that $m_{\text {sum }}^{2}$ remains fixed at $M^{2}$ for all times. In this case our system evolves only within the two-dimensional $(\alpha, \beta)$ plane shown in Fig. 2. Thus, for $\bar{m}_{\text {sum }}^{2}=M^{2}$ and any chosen $(\bar{\alpha}, \bar{\beta})$, the flow line connecting $(\alpha, \beta)=(1,1)$ to $(\alpha, \beta)=(\bar{\alpha}, \bar{\beta})$ indicates the path for the flow of our system as a function of time, with the corresponding values of $\lambda_{0}^{2}$ varying as the different $\lambda_{0}^{2}$-contour lines are crossed. This flow then terminates once the final location $(\bar{\alpha}, \bar{\beta})$ is reached. ${ }^{1}$

For any specified final location $(\bar{\alpha}, \bar{\beta})$, the results in Fig. 2 indicate how the lightest eigenvalue $\lambda_{0}$ varies as our system evolves in time. For our purposes, however, the most important feature of this dynamical evolution is the fact that $\lambda_{0}$ often evolves nonmonotonically, first rising and then falling again as we pass through the phase transition. Indeed, this nonmonotonicity in $\lambda_{0}$ occurs for all flow lines which cross the green line in Fig. 2, with values of $(\bar{\alpha}, \bar{\beta})$ lying below and/or to the left of this green line. As we see from Fig. 2, such behavior tends to be rather generic across much of the $(\bar{\alpha}, \bar{\beta})$ parameter space, and is particularly dramatic in cases for which $\bar{\alpha}<0$ and $\bar{\beta}$ is relatively small.

We can understand how this behavior arises as follows. At early times prior to the phase transition, the eigenvalues of our mass matrix are $\lambda_{0}=0$ and $\lambda_{1}=M$. However, as we

\footnotetext{
${ }^{1}$ As an aside, we note that since a given flow line is specified by the choice of its final location $(\bar{\alpha}, \bar{\beta})$, our ability to plot general flow lines without endpoints as in Fig. 2 rests upon a highly nontrivial fact: any flow line passing through a given point $\left(\alpha^{*}, \beta^{*}\right)$ en route to its final location $(\bar{\alpha}, \bar{\beta})$ entirely subsumes the (a priori distinct) flow line for which the final location is instead taken to be $\left(\alpha^{*}, \beta^{*}\right)$. In other words, all flow lines which begin at $(\alpha, \beta)=(1,1)$ and include a given intermediate point $\left(\alpha^{*}, \beta^{*}\right)$ actually coincide for all portions of the flow between these two points regardless of the particular time at which the $\left(\alpha^{*}, \beta^{*}\right)$ point is reached. Interestingly, this in turn implies that any arbitrary shift $t^{*} \rightarrow t^{*}+\delta t$ in the time $t^{*}$ at which a given intermediate point is reached can be realized simply by shifting the final location $(\bar{\alpha}, \bar{\beta})$ of the flow line (and thereby shifting the underlying theory). Moreover, this shift in $(\bar{\alpha}, \bar{\beta})$ simply moves $(\bar{\alpha}, \bar{\beta})$ along the same flow line. In this sense, then, these flow linesnow viewed as trajectories within the $(\bar{\alpha}, \bar{\beta})$ plane-have an additional interpretation as classical renormalization-group flows which describe how our different theories are connected under such time shifts. While this discussion has been limited to the special case with $m_{\text {sum }}^{2}=M^{2}$, as shown in Fig. 2, these properties hold even for flows that traverse the full three-dimensional $\left(m_{\text {sum }}^{2}, \alpha, \beta\right)$ parameter space.
}

enter the phase transition, the value of $\lambda_{0}$ inevitably begins to rise, and when $\bar{\alpha}<0$ the two eigenvalues $\lambda_{0,1}$ actually begin to approach each other. There is no level-crossing, however, because the mixing between these states ultimately induces a level repulsion which kicks $\lambda_{1}$ toward even higher values while causing $\lambda_{0}$ to drop again before settling into its final, asymptotic value. This level repulsion is particularly strong in cases for which the two states experience significant mixing (i.e., cases in which $\bar{\beta}$ is relatively small). Thus it is ultimately level repulsion that lies at the root of the nonmonotonicity of $\lambda_{0}$.

This nonmonotonic "pulse" behavior for $\lambda_{0}$ is sketched in Fig. 3. For concreteness, we have also superimposed a Hubble parameter which falls with time as $H(t) \sim 1 / t$. As we shall see, the existence of such a pulse has two important consequences. Both of these will turn out to be critical ingredients in building our eventual slingshot.

The first of these ingredients is the existence of a "re-overdamped" phase in the dynamics of the lighter scalar field. Recall that in general, a scalar of constant mass $m$ begins in an overdamped phase if $3 H(t) \geq 2 m$ at a sufficiently early time $t$. During such a phase, the field VEV remains approximately constant, with very little kinetic energy. However, since the Hubble parameter falls with time as the universe evolves, there eventually comes a point at which such a scalar transitions to an underdamped phase in which $3 H(t)<2 m$. The field VEV then begins to oscillate around zero. However, if the mass of the scalar in question experiences a pulse, as shown in Fig. 3, it is possible for the scalar to become overdamped once

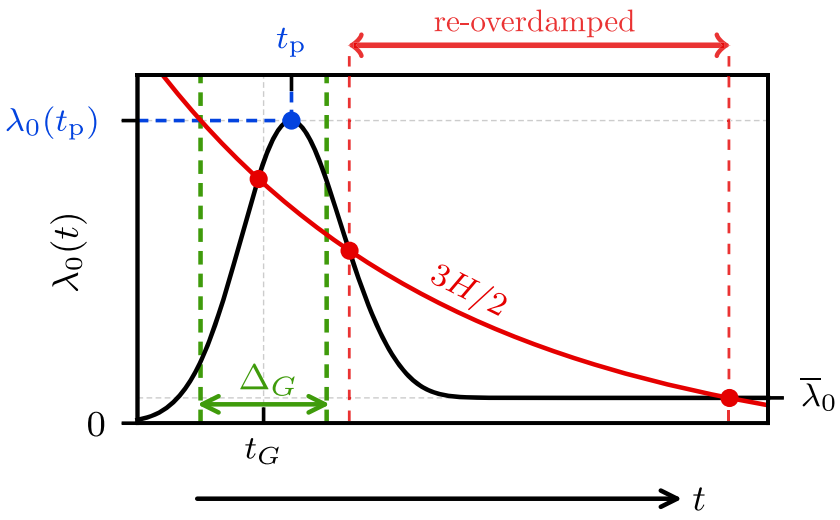

FIG. 3. "Pulse" behavior for the lightest eigenvalue $\lambda_{0}$, sketched as a function of time. This pulse behavior is ultimately the result of level repulsion between the two scalars in our system, and is particularly severe when the two mass eigenvalues approach each other (as occurs when $\bar{\alpha}<0$ ) and experience significant mixing (as occurs when $\bar{\beta}$ is relatively small). For the special case $m_{\text {sum }}^{2}=M^{2}$, such pulse behavior emerges for all $(\bar{\alpha}, \bar{\beta})$ lying below and/or to the left of the green contour in Fig. 2. As we shall see, this pulse behavior simultaneously gives rise to the two ingredients which are critical for building a slingshot for $\phi_{\lambda_{0}}$ : the phenomenon of re-overdamping and the possibility of a parametric resonance. 
again for a nontrivial interval of time-even after having already been underdamped. Indeed, we see from Fig. 3 that there are two distinct intervals of time during which $3 H(t) \geq 2 \lambda_{0}(t)$. Upon entering this second overdamped phase, the oscillations of the field VEV cease, as expected. However, because the field VEV is already oscillating when it enters the re-overdamped phase, it enters with an initial velocity which is no longer subject to the restoring forces that would have produced oscillations. Such a field VEV thus retains this initial velocity, subject only to the Hubble friction that eventually brings this velocity to zero.

The existence of a "pulse" for the mass $\lambda_{0}$ can also potentially give rise to our second important ingredient: a parametric resonance that acts during the initial underdamped phase-i.e., during the pulse itself. Recall that in general a harmonic oscillator experiences an $n$ th-order parametric resonance that magnifies the amplitudes of successive oscillations if the mass of the oscillator exhibits its own oscillatory behavior whose frequency is approximately $(2 / n)$ times the natural oscillator frequency, with $n \in \mathbb{Z}^{+}$. However, this is exactly what can occur here, since the pulse described above furnishes us with a changing mass. Of course, a true parametric resonance requires that the mass experience periodic oscillations, whereas in our case $\lambda_{0}$ experiences only a single pulse. However, such a pulse mimics half an oscillation, and it turns out that even this small segment of an oscillation is sufficient to induce a parametric resonance provided this pulse has an appropriate effective frequency $\omega_{\text {eff. }}$ In general, the effective frequency $\omega_{\text {eff }}$ of the pulse may be obtained from the curvature of $\lambda_{0}^{2}(t)$ near $t \approx t_{\mathrm{p}}$, where $t_{\mathrm{p}}$ is the time at which the pulse reaches its maximum height, and is given by [17]

$$
\omega_{\mathrm{eff}}^{2}=-\left.4 \frac{\ddot{\lambda}_{0}}{\lambda_{0}}\right|_{t=t_{\mathrm{p}}} .
$$

By contrast, the natural frequency of the oscillating scalar field near $t \approx t_{\mathrm{p}}$ is nothing but $\lambda_{0}\left(t_{\mathrm{p}}\right)$, since it is the mass of the field that drives the oscillations. We thus obtain a condition for the existence of an $n$ th-order parametric resonance [17]:

$$
\left.\left(\frac{\ddot{\lambda}_{0}}{\lambda_{0}^{3}}\right)\right|_{t=t_{\mathrm{p}}}=-\frac{1}{n^{2}}, \quad n \in \mathbb{Z}^{+} .
$$

Varying the width $\Delta_{G}$ of the phase transition induces variations in the value of the left side of this equation. Thus, there exists a discrete set of phase-transition widths $\Delta_{G}^{(n)}$ for which this resonance condition is satisfied. Assuming the width $\Delta_{G}$ of the phase transition matches one of these resonant widths $\Delta_{G}^{(n)}$, an $n$ th-order parametric resonance therefore enhances the size of the field oscillations near the end of the underdamped phase that immediately precedes re-overdamping.
Putting these two ingredients together, we now have the basic recipe for our slingshot that propels the lighter mass-eigenstate field $\phi_{\lambda_{0}}$ to a super-Planckian VEV. Choosing an appropriate set of parameters $(\bar{\alpha}, \bar{\beta})$ gives rise to a pulse for the mass $\lambda_{0}$ of this field. If this pulse has an appropriate shape, as described in Eq. (2.15), the field oscillates during the pulse. Of course, it is critical for the functioning of our slingshot that we enter the reoverdamped phase precisely at a moment where the oscillating field has its maximum velocity, so that our field is "released" from oscillatory behavior and launched with the maximum possible velocity. However, it turns out that the condition for this to happen is the same as that in Eq. (2.15) which establishes the parametric resonance in the first place. Thus, once the parametric resonance is established through the proper choice of $\Delta_{G}$, the resulting pulse has precisely the correct width in order to launch the field VEV with maximum velocity into the reoverdamped phase. Indeed, it turns out that if the width of the phase transition is given by $\Delta_{G}^{(n)}$, appropriate for satisfying the $n$ th-order parametric resonance condition, then our field experiences $n / 2$ oscillations within the underdamped phase before the eventual "launch" into the re-overdamped phase. As a result, parametric resonances with odd orders $n$ launch their field VEVs in a direction which is opposite to that for even orders $n$.

These points are illustrated in Fig. 4, which shows our slingshot mechanism in action. In this figure, we show the time evolution of the lighter field $\phi_{\lambda_{0}}$ when the phasetransition width $\Delta_{G}$ is set to $\Delta_{G}^{(n)}$ for $n=1,2, \ldots, 6$. In each case, our field begins with a VEV at $-M_{p}$ and undergoes $n / 2$ oscillations within the underdamped phase before being launched toward a field value whose magnitude vastly exceeds that of its initial value prior to the phase transition. Indeed, in each case the field is "launched" precisely upon entrance into the re-overdamped phase.

It is natural to refer to this mechanism for generating a large field VEV as a "slingshot." In ancient times, prior to the invention of rubber and other elastic materials, a slingshot of the David/Goliath variety was fashioned by attaching a projectile to a rope, twirling the rope around overhead with increasing speed, and then releasing the projectile at just the right moment so as to launch the projectile in the desired direction. Our slingshot mechanism is essentially the same: we too begin with an interval of periodic oscillations enhanced through a parametric resonance, followed by a "release" from the oscillatory behavior at just the proper moment so as to propel the projectile forward with maximum velocity. Indeed, the projectile in this instance is nothing but the field VEV, and its release from oscillations is nothing but the entrance into the reoverdamped phase. Likewise, the different higher-order resonances correspond to the different points at which the release can take place, with successive higher orders of 


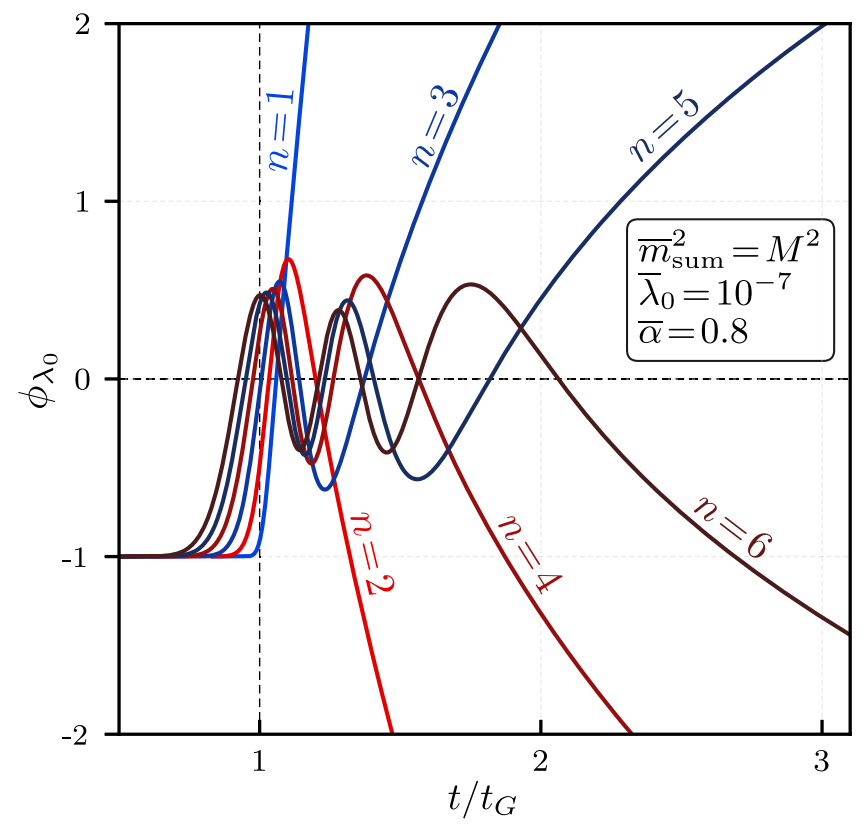

FIG. 4. The time-evolution of the $\phi_{\lambda_{0}}$ field in cases where the phase-transition width $\Delta_{G}$ is equal to a resonant width $\Delta_{G}^{(n)}$, plotted as functions of time for $1 \leq n \leq 6$ and assuming an initial field VEV $\phi_{\lambda_{0}}=-M_{\mathrm{P}}$ for $t \ll t_{G}$. (This sign is chosen as a convention so that the primary $n=1$ resonance propels our field VEV toward positive values.) For each $n$ we see that our slingshot mechanism successfully propels the field toward a large field VEV. In this figure (and in all subsequent figures in this paper), all dimensionful quantities are to be understood in units of appropriate powers of the Planck scale $M_{p}$, and we have taken $M=M_{p} / 3$ throughout. Note that the parameters chosen for this plot-namely $m_{\text {sum }}^{2}=M^{2}, \bar{\alpha}=0.8$, and $\bar{\lambda}_{0}=10^{-7} M_{p}$-correspond to the flow line indicated in red in Fig. 2.

resonance alternating between forward or backward motion of the projectile.

At first glance it might seem that our slingshot mechanism is fine-tuned in the sense that we must be precisely sitting on the parametric resonance, with $\Delta_{G}=\Delta_{G}^{(n)}$, in order to successfully launch our fields to other regions. However, this is not the case. In Fig. 5, we show the same $n=1$ and $n=2$ resonance curves from Fig. 4, along with 48 additional curves that illustrate the behavior of the field $\phi_{\lambda_{0}}$ when we are not sitting on either resonance, but rather are situated between these resonances. Indeed, in some sense these curves "interpolate" between the $n=1$ and $n=2$ resonance curves and correspond to phase-transition widths $\Delta_{G}$ which progress in equal-sized steps from $\Delta_{G}^{(1)}$ to $\Delta_{G}^{(2)}$. We see that even in cases which are off resonance, our slingshot mechanism continues to propel the field VEV toward large values. In other words, our parametric resonances are quite broad, and our system continues to benefit from the existence of these resonances even if it is not finely tuned to match their parameters.

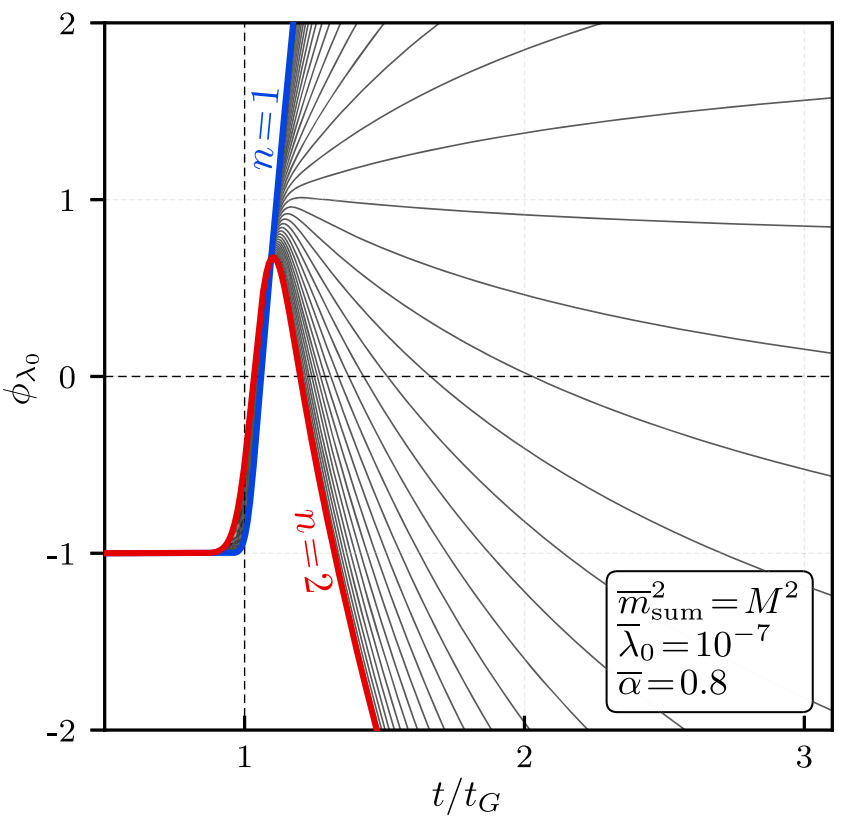

FIG. 5. The $n=1$ (blue) and $n=2$ (red) resonance curves from Fig. 4, along with a spectrum of 48 intermediate curves that describe the behavior of the $\phi_{\lambda_{0}}$ field for phase-transition widths $\Delta_{G}$ lying at equally spaced intervals between the resonant widths $\Delta_{G}^{(1)}$ and $\Delta_{G}^{(2)}$. From top to bottom, these curves correspond to phase-transition widths $\Delta_{G}=(1-r / 49) \Delta_{G}^{(1)}+(r / 49) \Delta_{G}^{(2)}$ with $r=1,2, \ldots, 48$ respectively. For reference, the curve whose asymptotic field behavior is almost completely flat corresponds to $r=27$. We see that even when we are not sitting precisely on a resonant width $\Delta_{G}^{(n)}$, our slingshot mechanism is often still capable of propelling our field VEVs to relatively high values. This ultimately happens because our parametric resonances are quite broad. Thus our slingshot mechanism does not require a significant amount of fine-tuning in order to operate as desired.

Given this slingshot mechanism, we can now proceed to consider how our scalar sector would evolve in a cosmological setting, both during and after the slingshot. In Fig. 6 we show the evolution of the masses $\lambda_{i}$ (top panel), the fields $\phi_{\lambda_{i}}$ (second panel), and their corresponding energy densities $\rho_{\lambda_{i}}$ (third panel). For all panels, the flow of time is indicated in terms of $\log \left(a / a_{G}\right) \equiv \log \left[a / a\left(t_{G}\right)\right]$, the number of $e$-folds since the phase transition, and we have plotted all of these quantities as functions of this number under our continuing assumption that our background cosmology is radiation-dominated. Note that the energy densities plotted in Fig. 6 are given by

$$
\bar{\rho}_{\lambda_{i}}=\frac{1}{2}\left(\dot{\phi}_{\lambda_{i}}^{2}+\bar{\lambda}_{i}^{2} \phi_{\lambda_{i}}^{2}\right)
$$

However, these energy densities can be meaningfully associated with the mass-eigenstate fields $\phi_{\lambda_{0}}$ and $\phi_{\lambda_{1}}$ only after the phase transition has effectively concluded and the mixing angle $\theta$ between these states has settled into its 

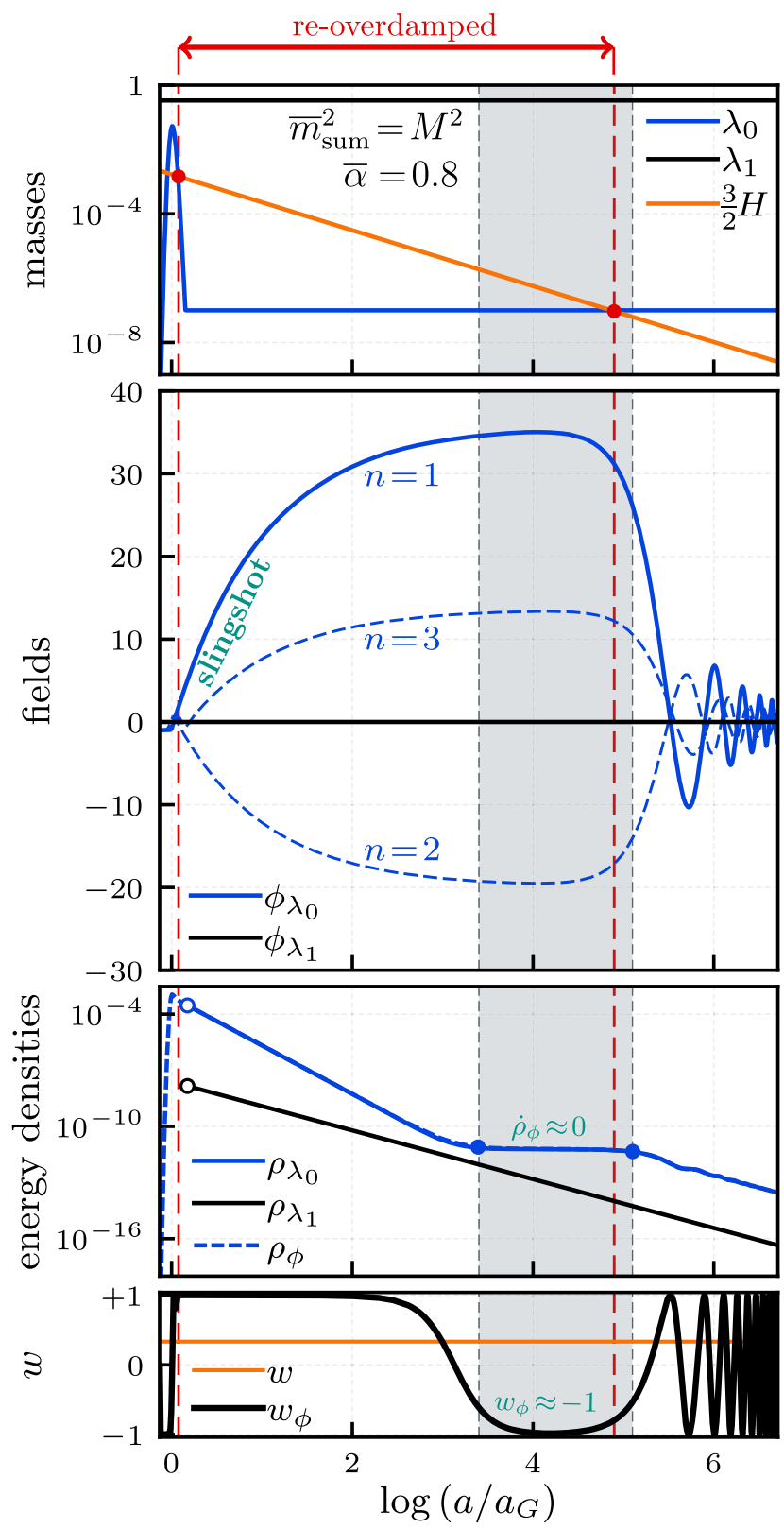

FIG. 6. Dynamical evolution of the scalar sector within a background (radiation-dominated) cosmology with a falling Hubble parameter $H(t) \approx 1 /(2 t)$. The different panels from top to bottom indicate the evolution of the scalar masses $\lambda_{i}$, the corresponding fields $\phi_{\lambda_{i}}$, their energy densities $\rho_{\lambda_{i}}$, and the equation-of-state parameter $w_{\phi}$ of the scalar sector. All curves correspond to the case of the $n=1$ resonance, except the dashed curves which show how the fields would have evolved under the $n=2$ and $n=3$ resonances instead. For this figure we have taken $M=M_{p} / 3$ and $\bar{\lambda}_{0}=10^{-7} M_{p}$ as reference values. We observe that $\dot{\rho}_{\lambda_{0}} \approx 0$ during the time interval shaded in gray, with the equation-of-state parameter for our scalar sector remaining near $w_{\phi} \approx-1$ during this interval.

asymptotic, late-time value $\bar{\theta}$. By contrast, at earlier times, the identities of the mass eigenstates are continually evolving and changing, and the existence of additional $\dot{\theta}$-dependent mixing terms within the total energy density $\rho_{\phi}$ of the scalar system prevents $\rho_{\phi}$ from being cleanly separated into individual contributions of the form appearing in Eq. (2.16). For this reason the separate energy densities $\rho_{\lambda_{i}}$ are plotted only after $\dot{\theta}$ has become sufficiently small and these contributions to $\rho_{\phi}$ can be meaningfully separated. The moment at which this occurs is indicated with open circles within this panel, and at earlier times we simply plot the total scalar-sector energy density $\rho_{\phi}$. Likewise, in Fig. 6 we have also plotted the equation-ofstate parameter $w_{\phi}$ of the scalar sector (bottom panel). This is defined as

$$
w_{\phi} \equiv \frac{P_{\phi}}{\rho_{\phi}}=\frac{\frac{1}{2} \sum_{k} \dot{\phi}_{k}^{2}-V_{\mathrm{eff}}\left(\phi_{i}, t\right)}{\frac{1}{2} \sum_{k} \dot{\phi}_{k}^{2}+V_{\mathrm{eff}}\left(\phi_{i}, t\right)},
$$

where $P_{\phi}$ and $\rho_{\phi}$ are the total pressure and energy density associated with the scalar scalar. For all panels of this figure we have chosen $M=M_{p} / 3$ as a benchmark value, with $m_{\text {sum }}^{2}=M^{2}, \bar{\alpha}=0.8$, and $\bar{\lambda}_{0}=10^{-7} M_{p}$. We have also taken our phase transition to have a width $\Delta_{G}=\Delta_{G}^{(1)}$.

The results in Fig. 6 can be understood as follows. In the top panel, we see the behavior of the mass eigenvalues $\lambda_{0,1}$ (blue and black curves, respectively), along with the Hubble curve superimposed (red). The Hubble parameter scales as $1 /(2 t)$, as expected for a radiation-dominated cosmology, while $\lambda_{1}$ remains close to $M_{p}$ across the full time interval shown in the figure. However $\lambda_{0}$ clearly exhibits the "pulse" we have discussed, along with a relatively long subsequent period of re-overdamping. This re-overdamping period is demarcated in Fig. 6 as that period existing between the vertical dashed red lines.

In the second panel, we show the corresponding behavior of the mass-eigenstate fields $\phi_{\lambda_{0,1}}$. As discussed above, the heavier field $\phi_{\lambda_{1}}$ (solid black curve) remains underdamped and thus experiences damped oscillations throughout the time interval shown, but the amplitudes of these oscillations are always sufficiently small that these oscillations are not readily evident in this figure. By contrast, the VEV of the lighter field $\phi_{\lambda_{0}}$ (solid blue curve) experiences a dramatic change as a result of the "slingshot" dynamics: this field is endowed with a huge velocity upon emerging from the phase transition and for this choice of model parameters is ultimately propelled to a value around $\phi_{\lambda_{0}} \approx 35 M_{p}$ after only a few $e$-folds. Indeed, it is only after the period of reoverdamping ends that this field begins to exhibit damped oscillations, as expected.

For purposes of comparison, we also display the corresponding $\phi_{\lambda_{0}}$ curves (dashed blue curves) that would result if we had taken $\Delta_{G}=\Delta_{G}^{(2)}$ or $\Delta_{G}=\Delta_{G}^{(3)}$ rather than $\Delta_{G}=\Delta_{G}^{(1)}$. We see that for each of these higher-order resonances, our slingshot mechanism likewise endows $\phi_{\lambda_{0}}$ 
with a large, trans-Planckian field VEV-albeit a VEV which is somewhat smaller than that obtained for the primary resonance.

In the third panel of Fig. 6, we show the evolution of the energy densities $\rho_{\lambda_{0,1}}$. The energy density $\rho_{\lambda_{1}}$ associated with the heavier scalar (black curve) declines steadily as $\rho_{\lambda_{1}} \propto a^{-3}$ across the entire time interval shown in the figure, as appropriate for a field which behaves like massive matter. By contrast, the energy density $\rho_{\lambda_{0}}$ of the lighter field (blue curve) evolves in nontrivial way. Immediately upon emerging from the phase transition, the energy density of this field is almost entirely kinetic. It thus redshifts much more rapidly, dropping as $\rho_{\lambda_{0}} \propto a^{-6}$. However, as the lighter field VEV approaches its apex, the dissipation of the corresponding energy density ceases almost entirely. Indeed, at this point the velocity of the lighter field approaches zero, whereupon our field is fully re-overdamped with no residual velocity remaining from the slingshot. This is thus an epoch in which the energy density of the lighter field behaves effectively as vacuum energy, with $\dot{\rho}_{\lambda_{0}} \approx 0$. Because of the importance of this epoch in our eventual discussion, we have shaded this $\dot{\rho}_{\lambda_{0}} \approx 0$ epoch with a gray background in Fig. 6. This epoch only ends when the period of re-overdamping ends, whereupon this field begins to oscillate again and $\rho_{\lambda_{0}}$ begins to fall accordingly as $1 / a^{3}$.

In the bottom panel of Fig. 6 we plot two quantities: the effective equation-of-state parameter $w_{\phi}$ for our scalar system (black curve), and the equation-of-state parameter $w$ for this entire example universe (orange). The behavior of $w_{\phi}$ follows directly from the properties we have seen in the previous panels. Because $\rho_{\lambda_{0}} \gg \rho_{\lambda_{1}}$ at all times after the phase transition, $w_{\phi}$ is essentially determined by the behavior of $\phi_{\lambda_{0}}$. At early times-i.e., for $a \ll a_{G}$-we have $w_{\phi} \approx-1$. However, the scalar sector becomes kineticenergy dominated, with $w_{\phi} \approx+1$, immediately after $\phi_{\lambda_{0}}$ is released from the slingshot. As we enter the fully reoverdamped region shaded in gray, $w_{\phi}$ drops to -1 , signifying the passage to a vacuum-energy dominated phase for the scalar sector. When the period of re-overdamping eventually ends and $\phi_{\lambda_{0}}$ begins to behave like massive matter, $w_{\phi}$ begins to oscillate around $w_{\phi} \approx 0$. Of course, despite this behavior for $w_{\phi}$, we see that the equation-ofstate parameter $w$ for the universe as a whole remains fixed at $w=+1 / 3$. This reflects our original assumption that we are operating within a background cosmology which is radiation-dominated, as consistent with the falling Hubble curve indicated in the top panel of Fig. 6 .

\section{BUILDING AN INFLATIONARY SLINGSHOT COSMOLOGY}

In this section, we discuss how the scalar sector and slingshot mechanism described in Sec. II can become the core elements of an inflationary cosmology. In Sec. III A, we describe how a full slingshot cosmology can be constructed and we demonstrate that such a cosmology can indeed give rise to an inflationary epoch. Then, in Sec. III B, we show that such an inflationary epoch can can have a duration sufficient to address the horizon and flatness problems.

\section{A. From scalar sector to inflationary slingshot cosmology}

In Sec. II, we considered a system of two scalar fields which undergo a cosmological phase transition and demonstrated a mechanism in which a parametric resonance followed by a re-overdamping phase together conspire to "slingshot" the VEV of the lighter field to super-Planckian values. We even found that such a system can give rise to many of the features normally associated with cosmological inflation, with the lighter scalar field effectively experiencing something akin to "slow-roll" behavior, with $\dot{\rho}_{\lambda_{0}} \approx 0$. Indeed, this situation arises within the gray shaded region in Fig. 6. We even demonstrated that the energy associated with our scalar sector as a whole behaves as vacuum energy during this period, with a corresponding equation-of-state parameter $w_{\phi} \approx-1$.

Unfortunately, this period of evolution is ultimately not inflationary. Inflation would require a slowly varying Hubble parameter $|\dot{H}| \ll H^{2}$, but instead our Hubble parameter is falling with $H(t) \approx 1 /(2 t)$, as consistent with our original assumption of a fixed radiation-dominated background cosmology. Likewise, we have seen that the equation of state for the entire universe is correspondingly fixed at $w=+1 / 3$, as expected under radiation-domination. Of course, in Sec. II we fixed the background cosmology in this way so that we could focus on the scalar sector. However, by embedding our scalar sector within a fixed background cosmology, we were effectively disregarding the gravitational backreaction of this cosmology on the scalar field dynamics. In other words, we were implicitly assuming that the scalar sector is only a subdominant component of our total cosmology, with a total scalar-sector energy density which is much less than the total energy density of the universe.

In order for our slingshot effect to potentially trigger an inflationary phase for the universe, we must instead enlarge our perspective by treating the scalar sector as the dominant component. However, this change in perspective immediately introduces several complications that must be considered.

First, we must take care to track the total energy flow within our cosmological setup. Recall that our scalar sector begins without energy, and only gains energy through the cosmological phase transition. Indeed, it is this phase transition which is ultimately responsible for the explicit time dependence in the effective potential $V_{\text {eff }}\left(\phi_{i}, t\right)$ in Eq. (2.2). However, if this energy is now to be considered 
the dominant component of energy density in the universe, then we must account for its source. In particular, since the energy density $\rho_{\phi}$ of our scalar sector has a time-evolution governed by

$$
\dot{\rho}_{\phi}+3 H\left(1+w_{\phi}\right) \rho_{\phi}=+\frac{\partial V_{\text {eff }}}{\partial t}
$$

there must also be a generic source sector with energy density $\rho_{S}$ governed by

$$
\dot{\rho}_{S}+3 H\left(1+w_{S}\right) \rho_{S}=-\frac{\partial V_{\mathrm{eff}}}{\partial t} .
$$

This will therefore balance the total energy budget in the $H \rightarrow 0$ limit (or equivalently in the co-moving frame), as required. It is of course natural to imagine that $w_{S}=-1$, so that this sector consists of vacuum energy, and we shall make this choice throughout the rest of this paper. Indeed, we shall further assume that $\rho_{S}$ is negligible after the phase transition, and thus its details are irrelevant for the physics after this transition.

In a similar vein, we may also wish to have a radiation sector of some sort to trigger the phase transition as the universe cools. Thus, we shall henceforth consider a minimal cosmological model consisting of three sectors: the scalar sector as outlined in Sec. II, a source sector as described above, and a radiation sector with total energy density $\rho_{R}$. Indeed, our discussion in Sec. II implicitly assumed the relation

$$
\rho_{S}+\rho_{\phi} \ll \rho_{R},
$$

but we now wish to consider the more general situation in which

$$
\rho_{S}+\rho_{\phi} \gtrsim \rho_{R}
$$

during and after the phase transition, when our slingshot operates. In fact, the specific initial conditions in Sec. II imply that $\rho_{\phi}=0$ prior to the phase transition, whereupon the assumption in Eq. (3.4) reduces to the assumption that $\rho_{S} \gtrsim \rho_{R}$.

This in turn leads to our second complication. In Sec. II, our analysis of the slingshot assumed a Hubble parameter which falls as $H(t) \approx 1 /(2 t)$, regardless of the dynamics of the scalar sector. However, if we now wish to incorporate the situation in Eq. (3.4), then the scalar and source sectors all contribute non-negligibly, and in general $H(t)$ is given by the Friedmann equation

$$
H=\sqrt{\frac{\rho_{\phi}+\rho_{S}+\rho_{R}}{3 M_{p}^{2}}} .
$$

This then introduces a gravitational back-reaction on the dynamics of scalar system, so that its equations of motion are rendered nonlinear. As a result, we expect the dynamics discussed in Sec. II to be significantly altered. In particular, we now must determine the extent to which the critical ingredients of our slingshot mechanism in Sec. II-e.g., the re-overdamping phase, the parametric resonance, etc.survive this gravitational backreaction. After all, it is a priori possible that our slingshot mechanism as a whole fails to survive this change. Moreover, even if these features do survive, it is possible that our parametric resonances might become much more narrow and thereby require an extreme fine-tuning in the value of $\Delta_{G}$. This too would be an undesirable outcome.

To investigate these possibilities, we repeat the calculations leading to Fig. 6, now under the assumption given in Eq. (3.4). In particular, we shall consider two particular cases of Eq. (3.4), one in which $\rho_{R}\left(t_{\star}\right)=M_{p}^{4}$ and the second in which $\rho_{R}\left(t_{\star}\right)=10^{-3} M_{p}^{4}$. We shall also continue to assume the same parameter choices as for Fig. 6, namely $M=M_{p} / 3, \bar{m}_{\text {sum }}^{2}=M^{2}, \bar{\alpha}=0.8$, and $\bar{\lambda}_{0}=10^{-7} M_{p}$.

Our results are shown in Fig. 7, where the solid (dotted) curves for $H(t), \rho_{R}(t)$, and $w(t)$ correspond to the larger (smaller) value of $\rho_{R}\left(t_{\star}\right)$ listed above. Several features immediately become apparent upon comparing these results with those in Fig. 6. First, we note from the top panel that the Hubble curve still crosses the lighter mass $\lambda_{0}$ at multiple points, giving rise to several damping transitions. In other words, the re-overdamping phase appears to survive gravitational backreaction. Second, examining the field evolution, we find that the parametric resonance also survives, including the higher-order resonances. Thus, our slingshot remains intact: we continue to have a relatively long period of re-overdamping at the beginning of which the VEV of the lighter field $\phi_{\lambda_{0}}$ is propelled to large, transPlanckian values. Indeed, we see that the period of reoverdamping is now significantly extended beyond what it was in Fig. 6, lasting for more than three times as many $e$-folds.

As before, the magnitude of the field VEV $\phi_{\lambda_{0}}$ continues to grow, in this case ultimately reaching a maximum value $\phi_{\lambda_{0}} \approx 8 M_{p}$, until the kinetic energy of the field is depleted by Hubble friction. This once again leads to an epoch during which $\dot{\rho}_{\lambda_{0}} \approx 0$, as shaded in gray. Indeed, during this epoch the lighter field satisfies the slow-roll conditions. Moreover, the energy density in the lighter scalar field continues to dominate that of the heavier scalar field, with $\rho_{\lambda_{0}} \gg \rho_{\lambda_{1}}$ throughout this interval. We therefore once again have $w_{\phi} \approx-1$.

However, in stark contrast to what occurs in Fig. 6, the total energy density for the universe is now dominated by the scalar field dynamics. Thus the transition to a vacuumdominated scalar sector now indicates the beginning of a truly inflationary epoch, with the lighter scalar field $\phi_{\lambda_{0}}$ 


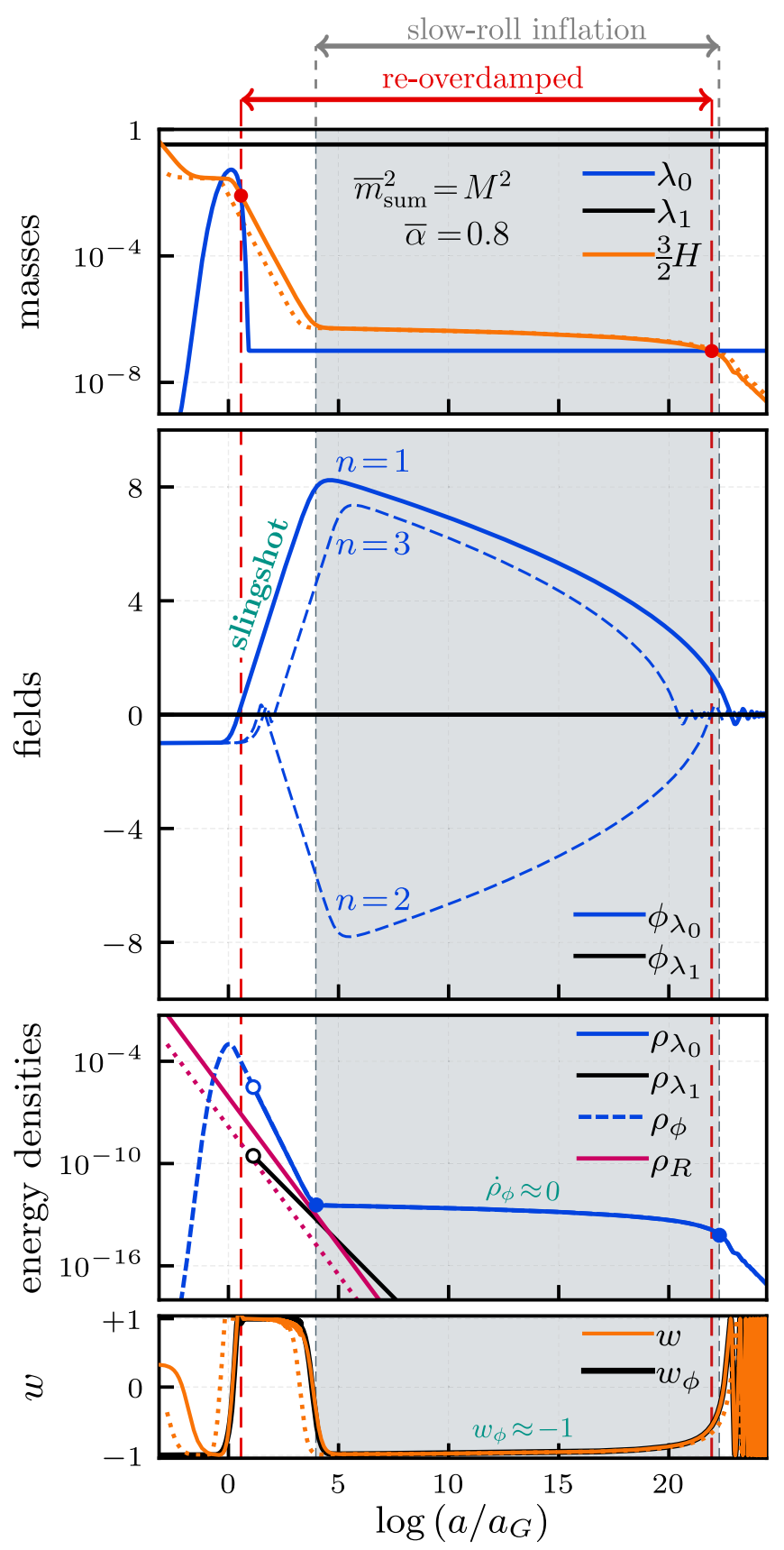

FIG. 7. Same as Fig. 6, except within the context of a cosmology in which the energy density of the scalar sector dominates. We see that the resulting gravitational backreaction not only preserves our slingshot but also leads to a period of slowroll inflation (shaded in gray).

serving as the inflaton. In other words, the epoch shaded in gray in Fig. 7 experiences slow-roll inflation, with $w \approx$ $w_{\phi} \approx-1$ throughout this epoch. This is consistent with the fact that the Hubble parameter in the top panel of Fig. 7 is now approximately constant during this period-the same fact which is responsible for lengthening the period of reoverdamping relative to what it was in Fig. 6, and thereby extending our period of inflation to a larger number of $e$ folds than would otherwise have occurred.

We thus conclude that an inflationary epoch can emerge from field configurations which, at first glance, do not lead to inflation. Indeed, it is the detailed properties of the cosmological phase transition which create the slingshot that propels the VEV of the inflaton field $\phi_{\lambda_{0}}$ to the superPlanckian values from which inflation then emerges.

The gravitational backreaction has additional important effects. For example, during the slingshot launch of the lighter scalar field $\phi_{\lambda_{0}}$, as the VEV of this field is growing toward its maximum value, the kinetic-energy density of this field dominates that of the scalar sector and thus dominates the Hubble damping. We thus find that $H \approx$ $\left|\dot{\phi}_{\lambda_{0}}\right| /\left(\sqrt{6} M_{p}\right)$, whereupon we see that $\phi_{\lambda_{0}}$ evolves linearly with respect to the number of $e$-folds $N_{\text {kin }} \equiv \int H d t$ during this period, i.e.,

$$
\frac{d \phi_{\lambda_{0}}}{d N_{\mathrm{kin}}} \approx \zeta \sqrt{6} M_{p}
$$

with an overall sign $\zeta \equiv(-1)^{n} \operatorname{sgn}\left(\mathcal{A}_{\phi}\right)$ which depends on the order $n$ of the resonance and the initial value $\mathcal{A}_{\phi}$ of $\phi_{0}$, as defined below Eq. (2.8). In this sense, the "velocity" $d \phi_{\lambda_{0}} / d N_{\text {kin }}$ of the field as it is launched is independent of the particular resonance involved. Likewise, we see that the maximum field VEV to which our lighter scalar field is launched is smaller in Fig. 7 than it was in Fig. 6. This loss of efficiency is not surprising, since the damping $H \sim \sqrt{\rho_{\phi}}$ is now directly determined by the amount of energy density in the scalar sector.

Consulting Fig. 7, we can also see the effect of varying $\rho_{R}\left(t_{\star}\right)$. As long as $\rho_{R}\left(t_{\star}\right) \lesssim M_{p}^{4}$, it turns out that $\rho_{R} \lesssim \rho_{\lambda_{0}}$ at the time when inflation begins. Thus the radiation component of the total energy density is already subdominant by this point, and changing $\rho_{R}$ will have essentially no effect on the resulting inflationary epoch. Of course, if $\rho_{R}$ were to exceed $\rho_{\lambda_{0}}$ at this time, then the onset of inflation would be delayed until the later time at which $\rho_{R}$ finally falls below $\rho_{\lambda_{0}}$. However, such a situation cannot happen because this greater value of $\rho_{R}$ would imply a super-Planckian radiation energy density prior to the phase transition, which is of course unphysical. The solid pink line for $\rho_{R}$ shown in Fig. 7 is therefore the maximum value for $\rho_{R}$ that can be adopted in any self-consistent cosmology. On the other hand, our choice for $\rho_{R}\left(t_{\star}\right)$ does have an effect on the physics prior to the phase transition, most notably as it concerns the overall equation-of-state parameter $w(t)$. However, all of these effects are transient, existing only for a short period of time until the phase transition sets in. We thus conclude that the physics of our inflationary epoch is largely independent of the particular subPlanckian choice for $\rho_{R}\left(t_{\star}\right)$, and we shall therefore 
adopt the simplifying assumption that $\rho_{R} \ll \rho_{S}+\rho_{\phi}$ in what follows.

We have not yet investigated the extent to which gravitational backreaction affects one important remaining feature of our slingshot mechanism. Recall that in the discussion surrounding Fig. 5, we observed that the slingshot is not especially fine-tuned in $\Delta_{G}$. However, it is possible - as with the other phenomena presented in Sec. II - that this property is spoiled by the gravitational backreaction. If so, this would be problematic, as such a fine-tuning would make it far less likely for realistic slingshot models to exhibit appropriate values of $\Delta_{G}$. However, this is ultimately not the case. In Fig. 8 we plot the maximum inflaton field VEV $\left|\phi_{\lambda_{0}}^{(\mathrm{I})}\right|$ that is ultimately reached after the slingshot as a function of the phasetransition width $\Delta_{G}$ for several different late-time eigenmasses $\bar{\lambda}_{0}$. As we see from Fig. 8, the parametric resonances generally continue to be extremely broad, a feature which remains valid regardless of the mass $\bar{\lambda}_{0}$ of the inflaton. In fact, each of the resonances in Fig. 8 is so broad that a fine-tuning is necessary in order to avoid resonant behavior. Our slingshot mechanism can therefore operate with a wide variety of phase-transition widths $\Delta_{G}$, and is

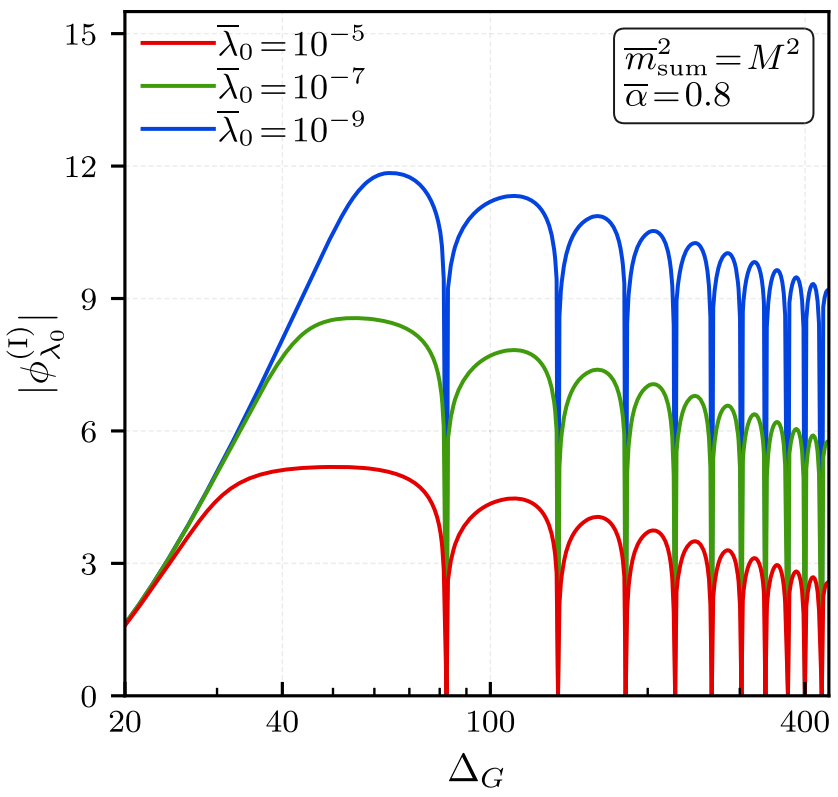

FIG. 8. The maximum field VEV $\left|\phi_{\lambda_{0}}^{(\mathrm{I})}\right|$ ultimately reached after the slingshot, plotted as a function of the phase-transition width $\Delta_{G}$ for several different late-time eigenmasses $\bar{\lambda}_{0}$. From left to right, the different peaks within each curve correspond to the successive higher-order parametric resonances driven by the mass-generating phase transition. We see that each of these peaks (particularly that associated with the left-most primary $n=1$ resonance) is quite broad, with essentially the same large VEV realized for a relatively large range of phase-transition widths $\Delta_{G}$ near their resonant values. Thus very little fine-tuning is required in order for our slingshot mechanism to capture the benefits of these parametric resonances. therefore not particularly sensitive to this aspect of the phase transition.

\section{B. Shooting further}

Thus far we have demonstrated that our slingshot mechanism-properly embedded within an appropriate cosmological framework-is capable of giving rise to an inflationary epoch. Indeed, given the parameters underlying the plots in Fig. 7, we have seen that the resulting inflationary epoch lasts for approximately $20 e$-folds. However, we have yet to explore the full parameter space of our model and thereby assess how many $e$-folds of inflation may ultimately be obtained through this mechanism. In particular, we seek to know whether we can exploit our slingshot mechanism in order to reach the target $N_{\text {inf }} \sim \mathcal{O}(50-60)$ needed to address the horizon and flatness problems.

Towards this end, we shall now perform a more systematic exploration of the parameter space of our slingshot model in order to identify the regions within which $N_{\text {inf }}$ is maximized. Our results are shown in Fig. 9. In this figure we display contours of the number $N_{\text {inf }}$ of $e$ folds of inflation produced by our slingshot, starting from $\phi_{\lambda_{0}}=\mathcal{A}_{\phi}=-M_{p}$ and $\dot{\phi}_{\lambda_{0}}=0$ prior to the phase transition. These results are obtained by numerically solving Eqs. (2.8) and (3.2) at each point in parameter space, with $H(t)$ given by Eq. (3.5). From left to right, the results shown in the three panels of the figure respectively correspond to the late-time inflaton-mass values $\bar{\lambda}_{0} / M_{p}=$ $\left\{10^{-5}, 10^{-7}, 10^{-9}\right\}$, and we have taken the width of the phase transition to be $\Delta_{G}=\Delta_{G}^{(1)}$ in each case.

Given the results in Fig. 9, we see that for each choice of $\bar{\lambda}_{0}$ there actually exists a global maximum for $N_{\text {inf }}$ within the $\left(\bar{\alpha}, \bar{m}_{\text {sum }}^{2}\right)$-space. The location of this maximum is largely insensitive to the value of $\bar{\lambda}_{0}$, and occurs around $\bar{m}_{\text {sum }}^{2} \approx M^{2}$ and $\bar{\alpha} \approx 0.9$ in each panel of this figure. We shall therefore adopt these as benchmark values for all future analyses in these paper. We also see that the global maximum value of $N_{\text {inf }}$ actually increases with decreasing $\bar{\lambda}_{0}$ - a fact already suggested in Fig. 8 on the basis of the corresponding values of $\left|\phi_{\lambda_{0}}^{(\mathrm{I})}\right|$ reached in each case.

The presence of such a global maximum for $N_{\text {inf }}$ stems from the interplay between two competing considerations. The first of these considerations is the value of the peak mass $\lambda_{0}\left(t_{\mathrm{p}}\right)$ at top of the pulse, as this determines the velocity with which the field is released from the slingshot. In order to indicate how $\lambda_{0}\left(t_{\mathrm{p}}\right)$ varies as a function of $\bar{m}_{\text {sum }}^{2} / M^{2}$ and $\bar{\alpha}$ for fixed $\bar{\lambda}_{0}$, we have also included within each panel of Fig. 9 contours (red curves) of the dimensionless ratio

$$
\mathcal{Q} \equiv \frac{\lambda_{0}\left(t_{\mathrm{p}}\right)}{\bar{\lambda}_{0}}
$$



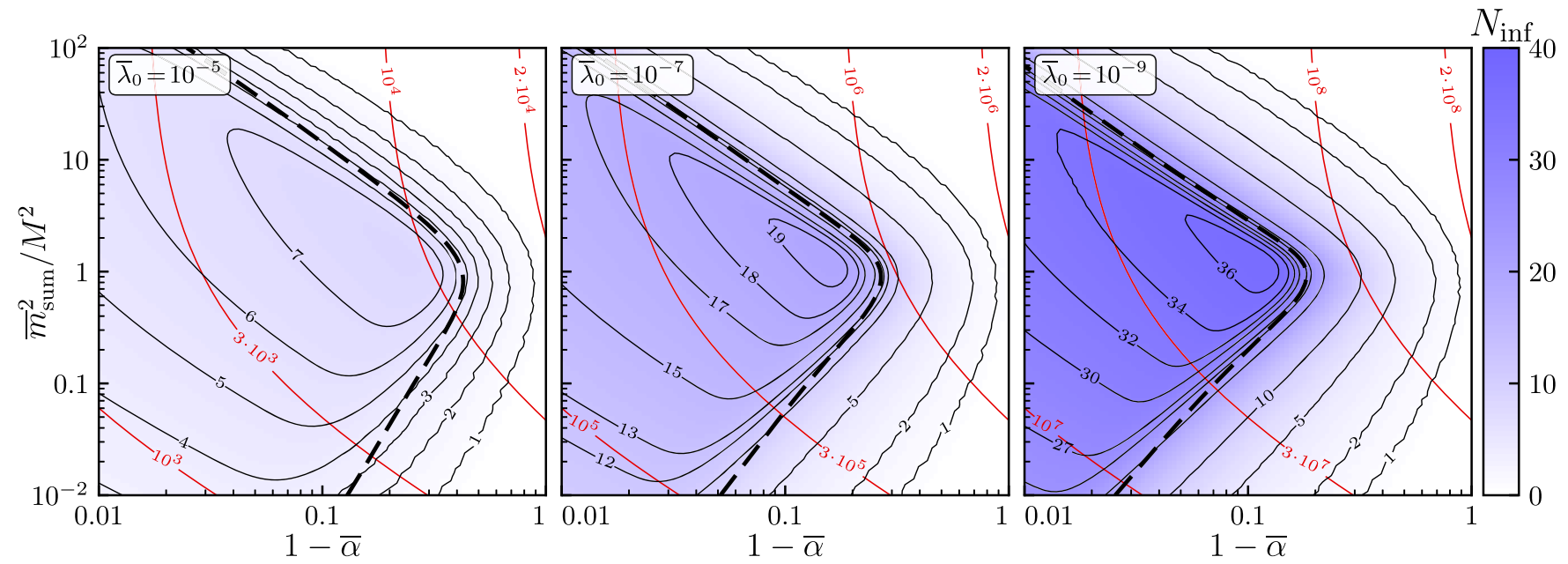

FIG. 9. Contours (black curves) showing the number of $e$-folds of inflation $N_{\text {inf }}$ produced by our slingshot, starting from $\phi_{\lambda_{0}}=$ $\mathcal{A}_{\phi}=-M_{p}$ and $\dot{\phi}_{\lambda_{0}}=0$, plotted within the $\left(\bar{\alpha}, \bar{m}_{\text {sum }}^{2}\right)$ plane assuming the primary $(n=1)$ resonance. The different panels correspond to different choices of the inflaton mass $\bar{\lambda}_{0}$. In each panel, the thick black dashed curve separates the regions in which the total energy density immediately prior to inflation is dominated by that of the lighter field (left) versus the heavier field (right). Contours of the mass quotient $\mathcal{Q} \equiv \lambda_{0}\left(t_{\mathrm{p}}\right) / \bar{\lambda}_{0}$ are also shown (red). In each case, we see that $N_{\text {inf }}$ is maximized immediately to the left of the thick black dashed curve, near $\bar{\alpha} \approx 0.9$ and $\bar{m}_{\text {sum }}^{2} \approx M^{2}$, and that this maximum value of $N_{\text {inf }}$ increases with decreasing inflaton mass $\bar{\lambda}_{0}$ (or increasing $\mathcal{Q}$ ).

Note that since $\bar{\lambda}_{0}$ is fixed within each panel, contours of $\mathcal{Q}$ are also essentially contours of $\lambda_{0}\left(t_{\mathrm{p}}\right)$. In general, we observe that within each panel, both $\mathcal{Q}$ and $\lambda_{0}\left(t_{\mathrm{p}}\right)$ increase with $\bar{m}_{\text {sum }}^{2} / M^{2}$ and with $1-\bar{\alpha}$ throughout the parameter space shown.

The second consideration which has a significant impact on the value of $N_{\text {inf }}$ is the energy density $\rho_{\lambda_{1}}$ of the heavier field. In the regions of parameter space within which $\rho_{\lambda_{1}}$ represents a significant fraction of $\rho_{\phi}$ at the onset of inflation, $N_{\text {inf }}$ is significantly suppressed. In order to illustrate the impact of this suppression, we have included a black dashed curve within each panel of Fig. 9 which separates the regions (to the left and right, respectively) in which either $\rho_{\lambda_{0}}$ or $\rho_{\lambda_{1}}$ dominates the other at the onset of inflation. We immediately see that $N_{\text {inf }}$ is significantly suppressed when $\rho_{\lambda_{1}}$ dominates. Thus, within each panel of the figure, we see that $N_{\text {inf }}$ increases with $\lambda_{0}\left(t_{\mathrm{p}}\right)$ (i.e., with $1-\bar{\alpha}$ and $\bar{m}_{\text {sum }}^{2} / M^{2}$ )—but only up to the point at which we cross this black dashed curve and this suppression sets in. It is for this reason that each value of $\bar{\lambda}_{0}$ or $\mathcal{Q}$ leads to a global maximum for $N_{\text {inf }}$.

Note that requiring that $\rho_{\lambda_{1}} \lesssim \rho_{\lambda_{0}}$ at the onset of inflation also places an upper bound on the initial energy density $\rho_{\lambda_{1}}$ (or equivalently $\rho_{1}$ ) at times $t_{\star}$ prior to the phase transition. To determine this bound analytically, we begin by noting that the mass of this field is sufficiently large that this field is always highly underdamped. Thus $\rho_{\lambda_{1}} \propto a^{-3}$ at all times other than during the phase transition. Moreover, relative to the timescales associated with the rapidly oscillating heavy field, the phase transition is essentially adiabatic and thus does not significantly perturb the time-evolution of this field away from this scaling behavior [assuming, of course, that the contribution to $\rho_{\lambda_{1}}$ generated by the phase transition is less than the initial $\rho_{\lambda_{1}}\left(t_{\star}\right)$ ]. In order to leave our inflationary epoch undisturbed, we therefore require that

$$
\rho_{\lambda_{1}}\left(t_{\star}\right) e^{-3 N} \lesssim \frac{1}{2} \bar{\lambda}_{0}^{2}\left[\phi_{\lambda_{0}}^{(\mathrm{I})}\right]^{2},
$$

where $N$ is the number of $e$-folds between $t_{\star}$ and the beginning of inflation. We can estimate $N$ by noting that the slingshot occurs approximately at the time $t_{G}+\Delta_{G}$, and therefore

$$
N \approx \frac{2}{3\left(1+\langle w\rangle_{\text {pre }}\right)} \log \left(\frac{t_{G}+\Delta_{G}}{t_{\star}}\right)+N_{\text {kin }},
$$

where $\langle w\rangle_{\text {pre }}$ represents a rough average value of the equation-of-state parameter $w(t)$ prior to the phase transition and where $N_{\text {kin }}$ represents the number of $e$-folds during the kination phase prior to inflation. Using an approximation for $N_{\text {kin }}$ to be derived in Eq. (4.2), we then find that Eq. (3.8) places a bound on the initial energy density:

$$
\rho_{\lambda_{1}}\left(t_{\star}\right) \lesssim \frac{1}{2} \bar{\lambda}_{0}^{2}\left(\frac{t_{G}+\Delta_{G}}{t_{\star}}\right)^{2 /\left(1+\langle w\rangle_{\text {pre }}\right)} \mathcal{Q}\left|\mathcal{A}_{\phi} \phi_{\lambda_{0}}^{(\mathrm{I})}\right| .
$$

Moreover, while a precise value of $\langle w\rangle_{\text {pre }}$ depends on model-specific details concerning our initial source and radiation sectors, we can obtain a conservative estimate by 
taking $\langle w\rangle_{\text {pre }}$ equal to its maximum value, which in this case is $+1 / 3$. Of course, the initial conditions we have chosen in Sec. II imply that $\rho_{\lambda_{1}}\left(t_{\star}\right)=0$, so the bound in Eq. (3.10) is always satisfied.

Overall, however, we see from Fig. 9 that our slingshot mechanism leads to an inflationary epoch spanning a significant number of $e$-folds within sizable regions of the parameter space shown. Moreover, comparing the results across the three panels of this figure, we also observe that the maximum value of $N_{\mathrm{inf}}$ is largest when $\bar{\lambda}_{0}$ is small and the corresponding value of $\mathcal{Q}$ at the location of the maximum is large.

Given this, the natural question is to determine how large $N_{\text {inf }}$ might become if we push this process still further. Unfortunately, due to numerical limitations, full contour plots of the sort shown in Fig. 9 become increasingly difficult to obtain as $\bar{\lambda}_{0}$ is taken increasingly small (or as $\mathcal{Q}$ is taken increasingly large). However, now that we have identified the location of parameter space in which $N_{\text {inf }}$ is maximized, and given that this region is largely independent of $\bar{\lambda}_{0}$, we can focus our numerical analysis to this smaller relevant region in order to study how the maximum value of $N_{\text {inf }}$ varies as a function of $\mathcal{Q}$.

Our results are shown in Fig. 10, where we plot the maximum field VEV $\left|\phi_{\lambda_{0}}^{(\mathrm{I})}\right|$ reached by our slingshot (top panel) as well as the corresponding number $N_{\text {inf }}$ of $e$-folds of inflation produced as functions of $\mathcal{Q}$ for several different initial conditions parametrized by $\mathcal{A}_{\phi}$ (solid colored curves). For this figure we have taken $M=M_{p} / 3, \bar{m}_{\text {sum }}^{2}=M^{2}$, $\bar{\alpha}=0.9$, and the primary resonance $\Delta_{G}=\Delta_{G}^{(1)}$. In all cases, we see that the maximum value of $N_{\text {inf }}$ can easily exceed our target range $N_{\text {inf }} \sim \mathcal{O}(50-60)$, provided $\mathcal{Q}$ is sufficiently large. Indeed, with these parameter choices the precise value $N_{\text {inf }}=60$ corresponds to $Q \approx 4 \times 10^{9}$ for $\left|\mathcal{A}_{\phi}\right|=M_{p}$, or equivalently $\bar{\lambda}_{0} \approx 10^{-11} M_{p}$. Thus, we conclude that our slingshot mechanism is indeed capable of yielding inflationary epochs of sufficient duration to solve the horizon and flatness problems - even when the inflaton starts with initial conditions from which inflation would not otherwise have been possible.

Interestingly, we also see from Fig. 10 that $N_{\text {inf }}$ does not grow without bound as $\mathcal{Q}$ is increased, but eventually saturates somewhat beyond our target range. This is apparently a direct result of the dynamics of this system. However, there is also another limitation on the size of $N_{\text {inf }}$. Increasing the value of $N_{\text {inf }}$ corresponds to increasing the abruptness with which our scalar-field eigenvalues $\lambda_{0,1}$ (and the mixings between the corresponding eigenstates) vary during the phase transition. Such changes render the evolution of the potential nonadiabatic, which in turn results in the production of particles and a concomitant loss of energy density from the zero-modes of $\phi_{\lambda_{0}}$ and $\phi_{\lambda_{1}}$. This too can ultimately suppress the maximum attainable value of $N_{\text {inf }}$. This particle-production effect and the

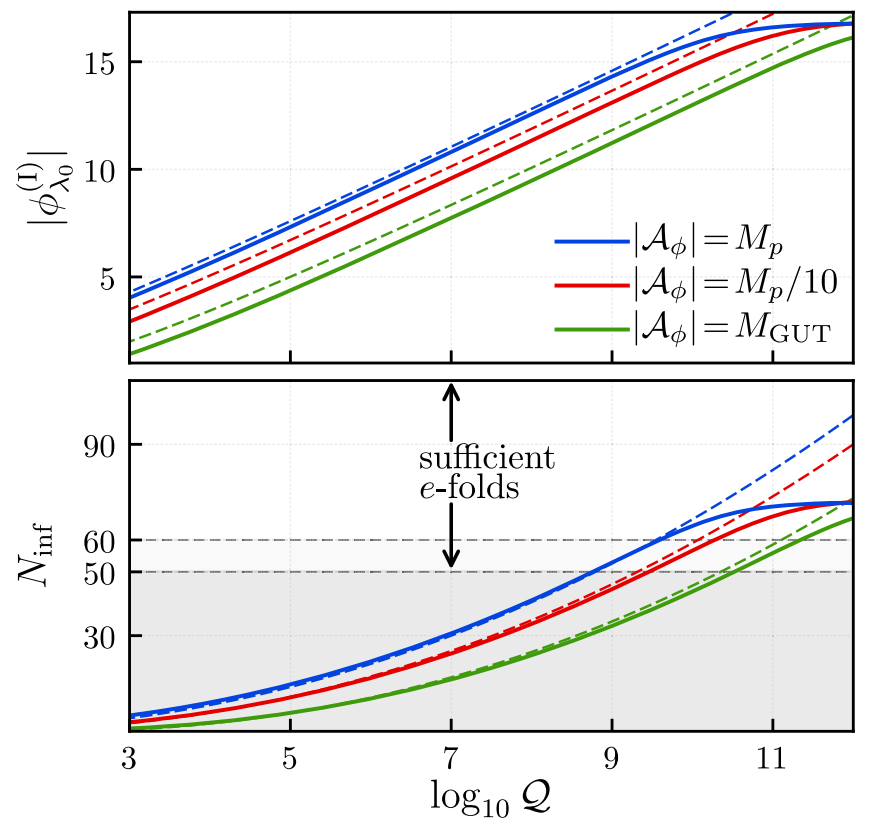

FIG. 10. The maximum field VEV $\left|\phi_{\lambda_{0}}^{(\mathrm{I})}\right|$ reached by our slingshot prior to the onset of inflation (top panel) as well as the corresponding number $N_{\text {inf }}$ of $e$-folds of inflation produced (lower panel), plotted as functions of $\mathcal{Q}$ for several different values of $\mathcal{A}_{\phi}$ assuming the primary $(n=1)$ resonance. The solid curves of a given color indicate the exact numerical values of $\left|\phi_{\lambda_{0}}^{(\mathrm{I})}\right|$ and $N_{\text {inf }}$ that result from numerically solving the evolution equations in Eqs. (2.8) and (3.2) in our preferred region of parameter space with $\bar{m}_{\text {sum }}^{2}=M^{2}$ and $\bar{\alpha}=0.9$, while the dashed curves indicate the results of analytical approximations to be discussed in Sec. IV. In each case, we conclude that for sufficiently large $\mathcal{Q}$, our slingshot mechanism is indeed capable of yielding inflationary epochs of sufficient duration to solve the horizon and flatness problems-even when the inflaton starts with initial conditions from which inflation would not otherwise have been possible.

corresponding upper bound it implies for $N_{\text {inf }}$ are discussed in greater detail in Appendix A. The upshot, however, is that our main conclusion is unchanged. Consequently our slingshot mechanism can indeed yield inflationary epochs of sufficient duration, as described above.

As we discussed in Sec. II, our slingshot mechanism has thus far been built on the assumption that $\phi_{0}\left(t_{\star}\right)=\mathcal{A}_{\phi}$ but that $\dot{\phi}_{0}\left(t_{\star}\right)=0$. A natural question, then, is to examine what might happen if we loosen this last restriction and consider arbitrary values of $\dot{\phi}_{0}\left(t_{\star}\right)$. In Fig. 11 we plot the resulting values of $N_{\text {inf }}$ as a function of $\dot{\phi}_{0}\left(t_{\star}\right)$ for a variety of different masses $\bar{\lambda}_{0}$. In this plot the solid and dashed lines respectively correspond to primary-resonance slingshots with $\rho_{R}\left(t_{\star}\right)=0.1 M_{p}^{4}$ and $\rho_{R}\left(t_{\star}\right)=10^{-3} M_{p}^{4}$, and we have taken $\mathcal{A}_{\phi}=M_{p}$ along with our usual benchmark choices $M=M_{p} / 3$ and $\bar{\alpha}=0.9$. Interestingly, when $\mathcal{A}_{\phi}$ and $\dot{\phi}_{0}\left(t_{\star}\right)$ are of opposite signs, our field is first propelled 


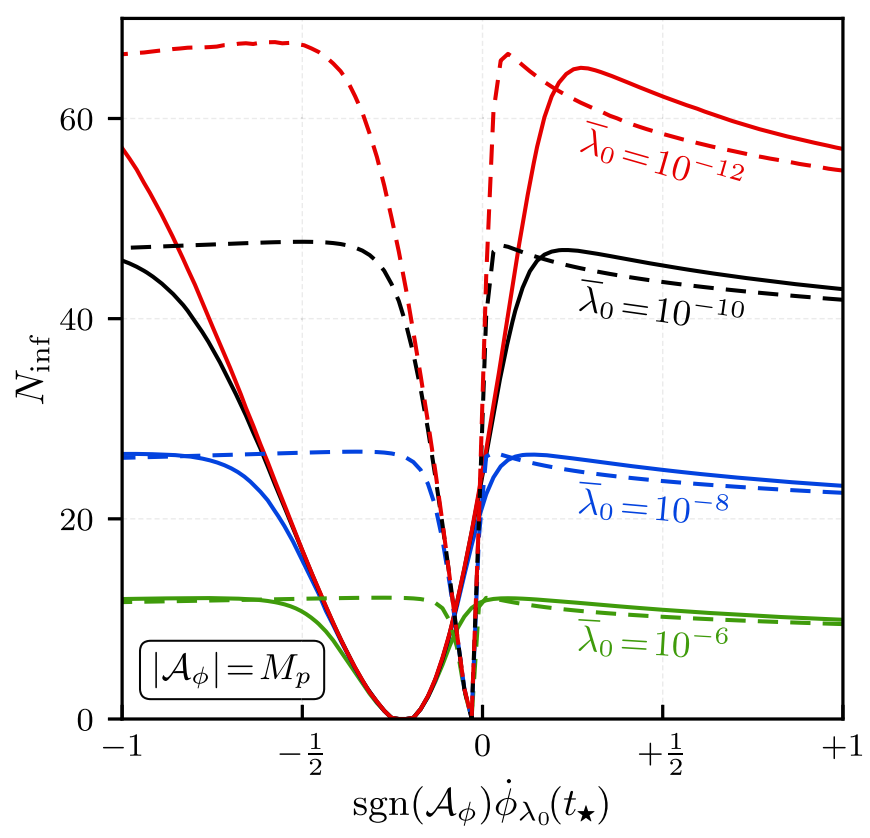

FIG. 11. The number of inflationary $e$-folds produced by our slingshot mechanism, plotted as a function of the initial field velocity $\dot{\phi}_{0}\left(t_{\star}\right)$ prior to the phase transition. The solid and dashed lines correspond to slingshots with $\rho_{R}\left(t_{\star}\right)=0.1 M_{p}^{4}$ and $\rho_{R}\left(t_{\star}\right)=10^{-3} M_{p}^{4}$, respectively. Despite the emergence of a strong localized suppression when $\mathcal{A}_{\phi}$ and $\dot{\phi}_{0}\left(t_{\star}\right)$ are of opposite signs, we see that $N_{\text {inf }}$ remains large and approximately constant when $\mathcal{A}_{\phi}$ and $\dot{\phi}_{0}\left(t_{\star}\right)$ are of the same sign. Thus our results are fairly insensitive to the precise value of $\dot{\phi}_{0}\left(t_{\star}\right)$ in such cases, and no fine-tuning of this variable is needed.

towards smaller field VEVs before ultimately experiencing the slingshot. This then results in a marked suppression in the total number of inflationary $e$-folds produced. Indeed, we see that the behavior of $N_{\mathrm{inf}}$ in the highly-suppressed region is largely independent of the mass $\bar{\lambda}_{0}$ of the inflaton. However, we also see from Fig. 11 that we can nevertheless achieve large values of $N_{\text {inf }}$ even when $\dot{\phi}_{0}\left(t_{\star}\right) \neq 0$. Indeed, when $\mathcal{A}_{\phi}$ and $\dot{\phi}_{0}\left(t_{\star}\right)$ are of the same sign, our results for $N_{\text {inf }}$ are approximately independent of $\dot{\phi}_{0}\left(t_{\star}\right)$, as claimed in Sec. II. Thus we do not need to fine-tune the value of $\dot{\phi}_{0}\left(t_{\star}\right)$ in order to achieve our results.

Finally, while the plots shown in Fig. 7 illustrate the cosmological evolution resulting from our slingshot mechanism, it is also instructive to understand our slingshot mechanism from the perspective of an inflaton phase-space diagram of the sort shown in Fig. 1. In Fig. 12 we show the same phase-space diagram as in Fig. 1 assuming the same quadratic inflaton potential $V\left(\phi_{\lambda_{0}}\right)=\frac{1}{2} m_{\phi}^{2} \phi^{2}$ where we now identify the inflaton field $\phi$ as $\phi_{\lambda_{0}}$ and the inflaton mass $m_{\phi}$ as $\bar{\lambda}_{0}$, and where we have now chosen $\bar{\lambda}_{0}=3 \times 10^{-12} M_{p}$. Superimposed on this diagram we also plot two possible inflaton trajectories: the first (yellow)

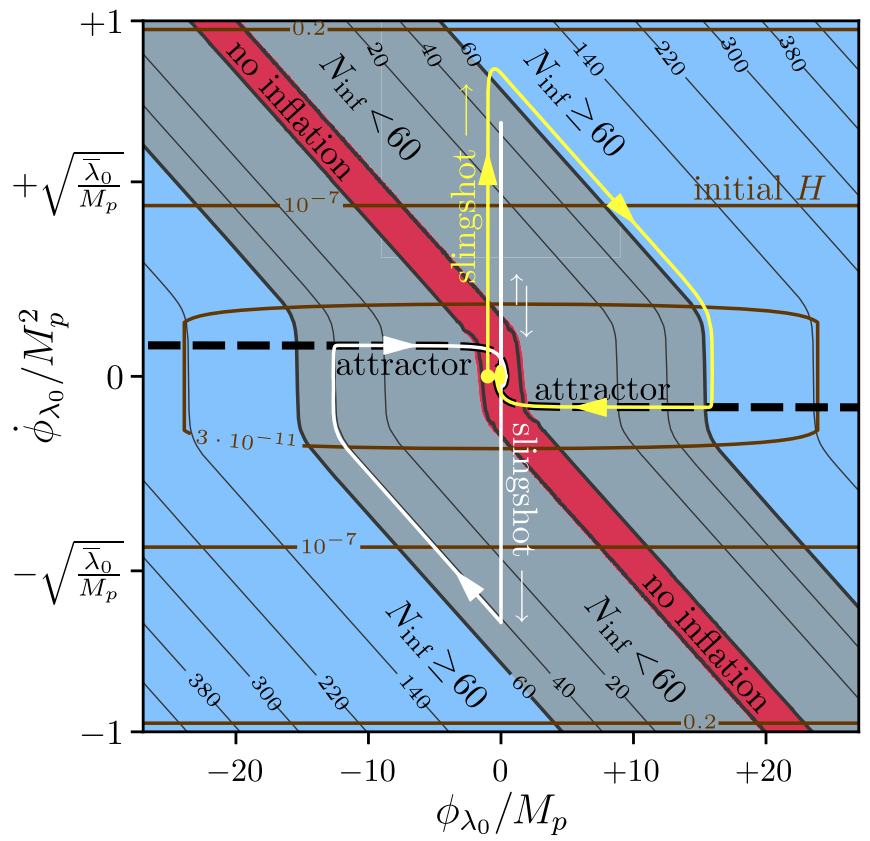

FIG. 12. The trajectory of the inflaton field $\phi_{\lambda_{0}}$ within the $\left\{\phi_{\lambda_{0}}, \dot{\phi}_{\lambda_{0}}\right\}$ phase space for different slingshots: the $n=1$ resonance with $\mathcal{A}_{\phi}=-M_{p}$ (yellow), and the $n=2$ resonance with $\mathcal{A}_{\phi}=-M_{\mathrm{GUT}}$ (white). Each slingshot is plotted for $\bar{m}_{\text {sum }}^{2}=M^{2}$, $\bar{\alpha}=0.9$, and $\bar{\lambda}_{0}=3 \times 10^{-12} M_{p}$. The background regions and contours are similar to those in Fig. 1, and are drawn for a quadratic inflaton potential $V\left(\phi_{\lambda_{0}}\right)=\frac{1}{2} \bar{\lambda}_{0}^{2} \phi_{\lambda_{0}}^{2}$. In the first case (yellow), our slingshot launches the inflaton from the red region upwards into the blue region, producing $N_{\text {inf }} \approx 65 e$-folds of inflation, while in the second case (white) our slingshot ultimately launches the inflaton from the red region downwards into the gray region, producing $N_{\text {inf }} \approx 42 e$-folds of inflation. Many other slingshot trajectories are also possible.

illustrates the slingshot trajectory with $\mathcal{A}_{\phi}=-M_{p}$ and $\Delta_{G}=\Delta_{G}^{(1)} \quad$ (corresponding to $\mathcal{Q} \approx 1.2 \times 10^{10}$ and $N_{\text {inf }} \approx 65$, in accordance with the results in Fig. 10), while the second (white) shows the trajectory with $\mathcal{A}_{\phi}=-M_{\mathrm{GUT}}$ and $\Delta_{G}=\Delta_{G}^{(2)}$ (corresponding to $N_{\text {inf }} \approx 42$ ), where $M_{\mathrm{GUT}} \approx 2 \times 10^{16} \mathrm{GeV}$. In each case all other parameters are chosen as in Fig. 10. The yellow and white inflaton trajectories each begin within the red region, signifying that their initial configurations would not ordinarily lead to inflation. However, in each case it is the slingshot mechanism which propels the inflaton into another phase-space region from which inflation can eventually emerge: in the first case the inflaton is propelled upwards into the blue region, while in the second case the inflaton is initially carried upwards but is then ultimately propelled downwards into the gray region-a reversal of direction which is consistent with the properties of the $n=2$ parametric resonance, as shown in Fig. 4.

In general, for a fixed inflaton potential, we have seen in Fig. 1 that any inflaton trajectory is restricted to follow 
contour lines of constant $N_{\text {inf }}$ until reaching an attractor. It may therefore seem surprising that our slingshot is capable of propelling our inflaton field across such contour lines into new regions of phase space which differ so fundamentally in their ability to generate inflation. However, as discussed in Sec. II, at the root of our slingshot mechanism is a cosmological phase transition which introduces a fundamental time dependence into our effective inflaton potential $V_{\text {eff }}\left(\phi_{i}, t\right)$. It is this time dependence which allows our inflaton trajectories to cross such contour lines. Phrased slightly differently, this explicit time dependence in the inflaton potential indicates that the phase transition induces a flow of energy into/out of the scalar sector, and it is this flow of energy which can kick the inflaton field to new locations in phase space. Additionally, this time dependence can endow the heavier scalar $\phi_{\lambda_{1}}$ with a non-negligible portion of the energy density, so that the energy density of the universe is not entirely dominated by that of the lighter field. However, after the phase transition has ultimately passed and the energy density of the heavier field has been diluted to negligible levels, our model is effectively described by a single light field $\phi_{\lambda_{0}}$ moving in a quadratic potential

$$
V\left(\phi_{\lambda_{0}}\right)=\frac{1}{2} \bar{\lambda}_{0}^{2} \phi_{\lambda_{0}}^{2},
$$

with $\phi_{\lambda_{0}}$ starting at the new phase-space location to which it has been propelled by the slingshot. Indeed, from this point forward, the inflaton will then follow the usual contour lines. Thus, it is only during the phase transition that the slingshot can work its magic, kicking the inflaton into a new region of phase space from which the standard inflationary dynamics then operates in order to produce inflation.

\section{MAPPING TO INFLATIONARY OBSERVABLES}

Having demonstrated the success of our slingshot mechanism in producing inflationary epochs of sufficient duration, we now turn to a somewhat more theoretical issue. Normally one would parametrize an inflationary model in terms of the VEV $\phi_{\lambda_{0}}^{(\mathrm{I})}$ of the inflaton at the moment when inflation commences. Indeed, the value of $\phi_{\lambda_{0}}^{(\mathrm{I})}$ in turn determines most of the quantities of interest which characterize the inflationary epoch, including $N_{\text {inf }}$. However, our slingshot mechanism and its associated phase transition furnish us with dynamics that occurs prior to this point. As such, this mechanism effectively establishes a map from parameters that characterize the phase transition to the inflaton VEV itself:

$$
\left\{M, \bar{m}_{i j}, \mathcal{A}_{\phi}, \Delta_{G}, t_{G}\right\} \rightarrow \phi_{\lambda_{0}}^{(\mathrm{I})}
$$

In this section, we shall construct this map analytically for the region of parameter space where $N_{\text {inf }}$ is a maximum, and then use this tool to elucidate different behaviors of the slingshot mechanism. These results will also be useful when we discuss several constraints on our mechanism in Sec. V. For concreteness, we shall focus on the case in which $n=1$, but we emphasize that qualitatively similar results are obtained for higher-order resonances as well.

In order to derive our analytic expression for $\phi_{\lambda_{0}}^{(\mathrm{I})}$, we begin by noting that $\phi_{\lambda_{0}}$ undergoes a period of underdamped oscillation during the phase transition, prior to the onset of re-overdamping. Throughout this period, $\phi_{\lambda_{0}}$ oscillates with a frequency approximately equal to the peak mass $\lambda_{0}\left(t_{\mathrm{p}}\right)$ which the inflaton attains as a result of the pulse. The velocity of the field as it is released by the slingshot is therefore approximately $\dot{\phi}_{\lambda_{0}} \sim \mathcal{A}_{\phi} \lambda_{0}\left(t_{\mathrm{p}}\right)$. Within our region of interest, the corresponding kineticenergy density $\frac{1}{2} \dot{\phi}_{\lambda_{0}}^{2} \sim \frac{1}{2} \mathcal{A}_{\phi}^{2} \lambda_{0}^{2}\left(t_{\mathrm{p}}\right)$ is sufficiently large that the universe enters a brief epoch of kination-domination immediately after this release, wherein $w \approx w_{\phi} \approx 1$.

During this epoch, however, the kinetic-energy density with which $\phi_{\lambda_{0}}$ is initially endowed is rapidly dissipated by Hubble damping, falling as $\rho_{\phi} \sim a^{-3\left(1+w_{\phi}\right)} \sim a^{-6}$ until essentially only potential energy remains. We can therefore determine the number of $e$-folds of kination-domination $N_{\text {kin }}$ by taking the ratio of $\rho_{\phi}$ between the beginning and ending of this period:

$$
e^{6 N_{\text {kin }}} \approx \frac{\frac{1}{2}\left[\lambda_{0}\left(t_{\mathrm{p}}\right) \mathcal{A}_{\phi}\right]^{2}}{\frac{1}{2}\left[\bar{\lambda}_{0} \phi_{\lambda_{0}}^{(\mathrm{I})}\right]^{2}} \approx \mathcal{Q}^{2} \frac{\mathcal{A}_{\phi}^{2}}{\left[\phi_{\lambda_{0}}^{(\mathrm{I})}\right]^{2}} .
$$

Here $\mathcal{Q}$ is the mass ratio defined in Eq. (3.7). Meanwhile, during the kination-dominated epoch, $\phi_{\lambda_{0}}$ evolves from approximately its initial value $\phi_{\lambda_{0}} \approx \mathcal{A}_{\phi}$ to its value $\phi_{\lambda_{0}}^{(\mathrm{I})}$ when inflation begins. Since the rate of change in $\phi_{\lambda_{0}}$ during the kination-dominated epoch is given by Eq. (3.6), and since we are focusing our attention on the $n=1$ resonance, we have

$$
\phi_{\lambda_{0}}^{(\mathrm{I})} \approx \mathcal{A}_{\phi}-\operatorname{sgn}\left(\mathcal{A}_{\phi}\right) \sqrt{6} M_{p} N_{\mathrm{kin}} .
$$

By eliminating $N_{\text {kin }}$ between Eqs. (4.2) and (4.3), we may obtain a rough estimate for the value $\phi_{\lambda_{0}}^{(\mathrm{I})}$. However, in order to refine this estimate, we incorporate a correction factor into Eq. (4.2) which compensates for the fact that this relation was derived within the approximation that the transitions into and out of the kination-dominated epoch are effectively instantaneous. This correction factor may be parametrized as a linear shift in $N_{\text {kin }}$ of the form $N_{\text {kin }} \rightarrow N_{\text {kin }}+\delta N_{\text {kin }}$. By comparing the results obtained from Eq. (4.2) with our numerical results, we find that 
$\delta N_{\text {kin }} \approx-1 / 3$. Incorporating this correction, we find that $\phi_{\lambda_{0}}^{(\mathrm{I})}$ is approximately

$$
\left|\phi_{\lambda_{0}}^{(\mathrm{I})}\right| \approx \sqrt{\frac{2}{3}} M_{p} W\left(\frac{2 \sqrt{3} \mathcal{Q}}{e^{\sqrt{\frac{3}{2} \mathcal{A}_{\phi} \phi}}\left|\mathcal{A}_{\phi}\right|} M_{p}\right),
$$

where $W(x)$ denotes the Lambert $W$-function and where we have approximated $2^{-3 / 2} e \approx 1$ in the argument of this function.

Remarkably, despite the complicated dynamics associated with the phase transition, Eq. (4.4) indicates that the initial value $\phi_{\lambda_{0}}^{(\mathrm{I})}$ for the inflaton field at the onset of inflation is principally determined by the value of $\mathcal{Q}$. In this sense, $\mathcal{Q}$ acts as a figure of merit which determines the efficacy of the slingshot mechanism. Moreover, given that $\mathcal{Q}$ is simply the ratio of the peak mass $\lambda_{0}\left(t_{\mathrm{p}}\right)$ to the late-time inflaton mass $\bar{\lambda}_{0}$, there exists a straightforward mapping from the parameters $\left\{M, \mathcal{A}_{\phi}, \bar{m}_{i j}, \Delta_{G}, t_{G}\right\}$ to $\mathcal{Q}$, and hence to $\phi_{\lambda_{0}}^{(\mathrm{I})}$. For example, within our region of interest, we have $\bar{\beta} \ll 1$. Within this regime, the late-time inflaton mass is given approximately by

$$
\bar{\lambda}_{0}^{2} \approx \frac{1}{2} \bar{m}_{\text {sum }}^{2}\left(1-\bar{\alpha}^{2}\right) \bar{\beta} .
$$

Likewise, we find that the mass $\lambda_{0}\left(t_{\mathrm{p}}\right)$ at the peak of the pulse is given by

$$
\lambda_{0}^{2}\left(t_{\mathrm{p}}\right) \approx \frac{\overline{\mathcal{M}}_{00}^{2}\left[1+\frac{\bar{m}_{\text {sum }}^{2}}{M^{2}}-2 \sqrt{\frac{\overline{\mathcal{M}}_{11}^{2}}{M^{2}}}\right]}{1+\frac{\bar{m}_{\text {sum }}^{2}}{M^{2}}\left(\frac{\bar{m}_{\text {sum }}^{2}}{M^{2}}-2 \bar{\alpha}\right)} .
$$

Since $\bar{\lambda}_{0} \propto \bar{\beta}^{1 / 2}$ in this regime, while $\lambda_{0}\left(t_{\mathrm{p}}\right)$ is independent of $\bar{\beta}$, we have the freedom to adjust these two mass scales independently of one another by varying the mixing saturation. Thus, we can obtain a large $\mathcal{Q}$-and thus, by extension, a large field VEV $\phi_{\lambda_{0}}^{(\mathrm{I})}$ - by taking $\bar{\beta}$ to be small and thereby arranging a separation between the mass scales $\lambda_{0}\left(t_{\mathrm{p}}\right)$ and $\bar{\lambda}_{0}$.

From the relationship in Eq. (4.4) between our model parameters and $\phi_{\lambda_{0}}^{(\mathrm{I})}$, it is straightforward to determine the number of $e$-folds of inflation generated by our slingshot mechanism. In particular, since the final value of $\phi_{\lambda_{0}}$ at the end of inflation is $\phi_{\lambda_{0}}^{(\mathrm{E})}=\sqrt{2} M_{p}$, we find that

$$
N_{\mathrm{inf}} \approx \int_{\phi_{\lambda_{0}}^{(\mathrm{I})}}^{\phi_{\lambda_{0}}^{(\mathrm{E})}} d \phi_{\lambda_{0}} \frac{H}{\dot{\phi}_{\lambda_{0}}} \approx \frac{1}{4} \frac{\left[\phi_{\lambda_{0}}^{(\mathrm{I})}\right]^{2}}{M_{p}^{2}}-\frac{1}{2},
$$

with $\phi_{\lambda_{0}}^{(\mathrm{I})}$ given by Eq. (4.4). We may simplify the resulting expression by noting that $\mathcal{Q} \gg M_{p} /\left|\mathcal{A}_{\phi}\right| \gtrsim 1$ within our region of interest and invoking the approximation $W(x) \approx$ $\log (x / \log x)$ for $x \gg 1$. We then find that

$N_{\text {inf }} \approx \frac{1}{6} \log ^{2}\left[\frac{2\left|\mathcal{A}_{\phi}\right| \mathcal{Q} e^{\sqrt{\frac{3}{2}}\left(1-\frac{\left|\mathcal{A}_{\phi}\right|}{M_{p}}\right)}}{1+\frac{3}{\sqrt{2}}\left(1-\frac{\left|\mathcal{A}_{\phi}\right|}{M_{p}}\right)+\sqrt{3} \log \left(\frac{\left|\mathcal{A}_{\phi}\right|}{M_{p}} \mathcal{Q}\right)}\right]$.

In Fig. 10, we have already plotted the exact values of $\left|\phi_{\lambda_{0}}^{(\mathrm{I})}\right|$ and $N_{\text {inf }}$ as functions of $\mathcal{Q}$ (solid curves) using results obtained through full numerical calculations. We now superimpose on these plots the results of the analytic approximations in Eqs. (4.4) and (4.8) for $\left|\phi_{\lambda_{0}}^{(\mathrm{I})}\right|$ and $N_{\text {inf }}$ respectively (dashed curves). As we see from this figure, these two sets of results coincide well for all $\mathcal{Q} \lesssim 10^{10}$. We thus conclude that Eqs. (4.4) and (4.8) provide excellent approximations for $\left|\phi_{\lambda_{0}}^{(\mathrm{I})}\right|$ and $N_{\text {inf }}$ as functions of $\mathcal{Q}$ for all $\mathcal{Q}$ within this range. Most importantly, this includes the target range for $N_{\text {inf }}$ in which we are most interested, namely that for which $N_{\text {inf }} \sim \mathcal{O}(50-60)$. Of course, these approximations fail to capture the suppression for $\left|\phi_{\lambda_{0}}^{(\mathrm{I})}\right|$ and $N_{\text {inf }}$ that develops for $\mathcal{Q} \gtrsim 10^{10}$. This is not unexpected, as this suppression involves effects beyond those involved in this analytical approximation.

\section{CONSTRAINTS}

At this point we have realized our main objectives in this paper: we have developed a "slingshot" mechanism, we have shown that it can lead to an inflationary epoch, and we have further shown that there exist regions of parameter space wherein a sufficient number of $e$-folds arises. In this final section, we shall take a cursory look beyond these primary objectives and briefly discuss the observational consequences of this particular realization of the slingshot mechanism.

Generally speaking, most of the useful information we have about the inflationary epoch is derived from the spectrum of anisotropies detected in the CMB. Since $\bar{\lambda}_{0} \ll H$ during the inflationary epoch, our inflaton field $\phi_{\lambda_{0}}$ experiences quantum fluctuations

$$
\delta \phi_{\lambda_{0}} \approx \frac{H}{2 \pi}
$$

throughout this epoch. These fluctuations are systematically "frozen-in" as classical curvature perturbations while the universe inflates. Specifically, a fluctuation with comoving wavenumber $k$ effectively becomes classical once $k<a H$ and the corresponding wavelength exceeds the comoving horizon. To a zeroth-order approximation these primordial perturbations are scale-invariant, since $H$ is approximately constant during inflation. It is therefore sensible to parametrize the scalar power spectrum $\mathcal{P}_{s}(k)$ and tensor power spectrum $\mathcal{P}_{T}(k)$ in terms of their 
deviations from a purely scale-invariant form [19]. In particular, these primordial power spectra are typically parametrized as

$$
\begin{aligned}
& \mathcal{P}_{s}(k)=A_{s}\left(\frac{k}{k_{*}}\right)^{n_{s}-1}, \\
& \mathcal{P}_{T}(k)=A_{T}\left(\frac{k}{k_{*}}\right)^{n_{T}},
\end{aligned}
$$

where $A_{s}$ and $A_{T}$ are the respective amplitudes and $n_{s}, n_{T}$ are the scalar and tensor spectral indices. The fiducial wavenumber $k_{*}$ which appears in both $\mathcal{P}_{s}(k)$ and $\mathcal{P}_{T}(k)$ corresponds to the scale of perturbations which exit the horizon some $N_{\text {inf }}^{*} e$-folds before the end of inflation. Since these modes leave detectable imprints in the CMB, observational data constrains the values of the parameters $A_{s}, A_{T}$, $n_{s}$, and $n_{T}$ and combinations thereof.

While the slingshot dynamics which leads to the inflationary epoch is fairly complicated, the corresponding predictions for inflationary observables are relatively straightforward if $N_{\text {inf }} \gg N_{\text {inf }}^{*}$. The reason is that once our phase transition has concluded, the inflaton potential in this model is static and purely quadratic in $\phi_{\lambda_{0}}$. For a potential of this form, most inflationary observables are effectively determined by the value of $N_{\text {inf }}^{*}$ alone, provided that $N_{\text {inf }} \gg N_{\text {inf }}^{*}$. For example, the ratio of scalar-to-tensor power is given by

$$
r \equiv A_{s} / A_{T} \approx \frac{16}{1+2 N_{\mathrm{inf}}^{*}},
$$

while the scalar spectral index is given by

$$
n_{s} \approx 1-\frac{2}{1+2 N_{\mathrm{inf}}^{*}},
$$

and so forth. In other words, the observational constraints which apply to our model are essentially the same as those which apply to other models of inflation in which the inflaton potential is purely quadratic, regardless of the complicated dynamics through which the initial conditions for the inflaton field are established.

By contrast, the amplitude $A_{s}$ in Eq. (5.2) depends not solely on $N_{\text {inf }}^{*}$, but on other model parameters as well. In particular, $A_{s}$ is in determined by the energy scale of inflation-or equivalently, given our potential, the late-time mass $\bar{\lambda}_{0}$ of the inflaton-through the relation

$$
\begin{aligned}
A_{s} & =\left.\frac{1}{12 \pi^{2} M_{p}^{6}}\left[\frac{V^{3 / 2}\left(\phi_{\lambda_{0}}\right)}{V^{\prime}\left(\phi_{\lambda_{0}}\right)}\right]^{2}\right|_{\phi_{\lambda_{0}}=\phi_{\lambda_{0}}^{*}} \\
& =\frac{1}{6}\left(\frac{\bar{\lambda}_{0}\left[\phi_{\lambda_{0}}^{*}\right]^{2}}{4 \pi M_{p}^{3}}\right)^{2}
\end{aligned}
$$

where $\phi_{\lambda_{0}}^{*}$ is the inflaton VEV at the time at which the mode with wavenumber $k_{*}$ exits the horizon. Recent measurements by the Planck Collaboration at the scale $k_{*}=$ $0.05 \mathrm{Mpc}^{-1}$ yield an amplitude $A_{s} \approx 2 \times 10^{-10}$ [20] for scalar perturbations. Thus, in order to produce both sufficient scalar power and a sufficient amount of inflationary expansion, the inflaton mass must reside around the scale $\bar{\lambda}_{0}=\mathcal{O}\left(10^{-6} M_{p}\right)$ in such a scenario.

If we assume that the dominant primordial perturbations are generated by quantum fluctuations of the inflaton field, then a tension clearly exists between Eq. (5.5) and other predictions which follow from this realization of our slingshot mechanism. Of course, a significant feature of this mechanism is that the initial inflation-triggering field configuration $\phi_{\lambda_{0}}^{(\mathrm{I})}$ is not put in by hand, but rather determined by the pre-inflationary phase transition. Indeed, our slingshot mechanism provides a linkage between $\bar{\lambda}_{0}$ and the field VEV $\phi_{\lambda_{0}}^{(\mathrm{I})}$ at the beginning of inflation, such that a degree of freedom is mapped from the inflation model to the phase transition.

This tension can be addressed in several ways. For example, one might consider a generalization of our minimal model in which the contributions to $V_{\text {eff }}(\phi, t)$ from the phase transition include not only quadratic terms, but higher-order terms as well. Alternatively, the primordial perturbations may originate from another sector, such as from the dynamics of a curvaton field $\chi$ [21-23], which couples negligibly to the scalars involved in our slingshot mechanism. Indeed, curvatons are a standard ingredient in many models of early-universe dynamics, and in our case the degrees of freedom associated with such a curvaton sector allow us to relieve this tension and thereby render our slingshot mechanism consistent with the observed perturbation spectrum. Although at early times the curvaton would be subdominant $\rho_{\chi} \ll \rho_{\phi}$ and thereby act as a spectator to the inflationary dynamics, the curvaton is still subject to fluctuations $\delta \chi \approx H /(2 \pi)$, provided it is sufficiently light. After inflation ends, the curvaton grows closer to dominating the energy density and eventually decays, transferring its perturbations to the radiation bath [24,25]. The properties of the resulting perturbation spectrum are then set not only by the Hubble scale during inflation, but also by details of the curvaton evolution. Furthermore, we note that while the curvaton dynamics depends on the inflation model, the slingshot mechanism we have discussed in this paper is not affected by the introduction of the curvaton. In general, the curvaton could conceivably have many different types of potentials which lead to very different field evolutions. Of course, for the (quadratic) inflaton potential discussed in this paper, concave-up curvaton potentials would fall short phenomenologically since they cannot produce a sufficiently red-tilted spectrum. However, as explicitly shown in Ref. [26] and then argued more generally in Ref. [27], potentials with a 
concave-down region can be phenomenologically viable. Thus, our slingshot mechanism is not only capable of producing an inflationary epoch with a sufficient number of $e$-folds but can also co-exist with a curvaton sector that helps to produce a phenomenologically acceptable perturbation spectrum.

We emphasize that the results of this section rests on the assumption that $N_{\text {inf }} \gg N_{\text {inf }}^{*}$. If this assumption does not hold, the analysis of the perturbation spectrum becomes more subtle. Indeed, in this case $\mathcal{P}_{s}(k)$ and $\mathcal{P}_{T}(k)$ ultimately depend on the state of the universe prior to inflation. Such considerations shall be discussed in greater detail in Sec. VI.

\section{CONCLUSIONS AND DISCUSSION}

In this paper, we have proposed a novel "slingshot" mechanism which can enlarge the space of viable initial field configurations for inflation. We have illustrated this mechanism within the context of a minimal model whose core is a sector consisting of two scalar fields undergoing a mass-generating phase transition. However, while this minimal model contains all of the essential ingredients necessary for our slingshot mechanism and yields a sufficient number of $e$-folds of inflation, there are a number of possible generalizations and extensions which merit further study.

One such possible generalization concerns the form of our effective potential $V_{\text {eff }}\left(\phi_{i}, t\right)$. For simplicity, we have focused in this paper on the case in which $V_{\text {eff }}\left(\phi_{i}, t\right)$ is quadratic in the fields of the scalar sector. However, in the most general case, higher-order terms may also arise in $V_{\text {eff }}\left(\phi_{i}, t\right)$. The presence of such anharmonic terms in the potential can have a significant impact on the scalar potential at large distances in field space away from its minimum, potentially giving rise to plateau regions or other features which could potentially alter the inflationary dynamics and impact inflationary observables.

One natural way in which such higher-order terms in the scalar potential might be generated is through a nonminimal coupling of the fields in the scalar sector to gravity. In particular, one could consider scenarios in which a nonminimal coupling between the $\phi_{i}$ and the scalar curvature $\mathcal{R}$-e.g., a term $\propto \mathcal{R} \phi_{i}^{2}$-is present in the Jordan frame [28]. In such scenarios, the resulting modification of the scalar potential in the Einstein frame can help to ease tensions with applicable phenomenological constraints. Indeed, even in the simplest case in which the scalar potential is quadratic in the Jordan frame, a nonminimal coupling between the inflaton and $\mathcal{R}$ can deform the potential such that it becomes concave down at large field values, thereby rendering such a potential viable for realistic inflation [29,30]. It would therefore be interesting to investigate whether the introduction of such nonminimal couplings between the $\phi_{i}$ and $\mathcal{R}$ in the context of our slingshot mechanism would have similar advantages, enhancing the number of $e$-folds, while at the same time producing a sufficient amount of power in scalar perturbations.

An interesting feature of our slingshot mechanism is that the maximum number of $e$-folds of inflation obtained is only slightly above the threshold value $N_{\text {inf }} \approx \mathcal{O}(50-60)$ needed in order to solve the horizon and flatness problems. In most inflationary scenarios, the epoch of inflationary expansion typically begins long before currently observable modes in the perturbation spectrum exit the horizon. In such models, the Bunch-Davies vacuum [31,32] provides a natural set of initial conditions for these perturbations. By contrast, in scenarios with $N_{\text {inf }} \approx \mathcal{O}(50-60)$, the initial conditions which determine the spectrum of large-scale perturbations differ from those associated with the BunchDavies vacuum. In particular, they depend on the state of the universe prior to the inflationary epoch [33,34]. In the context of our slingshot mechanism, this spectrum in principle depends on the state of the universe both during the phase transition itself and during the kinationdominated phase that follows it. Thus, in scenarios which make use of our slingshot mechanism, the predictions for a number of inflationary observables could deviate from those associated with more standard inflationary scenarios. It would be interesting to investigate the possible perturbation spectra that can arise as a consequence-especially since the spectra associated with nonstandard initial conditions can exhibit certain features which are advantageous from a phenomenological perspective. For example, many scenarios involving other kinds of modifications of the cosmological history prior to inflation-among them fast-roll inflation [35-37], inflection-point inflation [38], "climbing-scalar" scenarios in braneworld cosmologies [39-42], other inflationary scenarios in string theory [43], and scenarios involving an early epoch of radiation-domination prior to inflation $[44,45]$ - have been invoked as explanations for the suppression of power at low multipole number in the CMB.

Detectable cosmological imprints of our slingshot mechanism could also potentially arise as a result of the nonadiabatic evolution of the fields within our scalar sector. One consequence of such nonadiabaticity is, of course, particle production due to rapid changes in the inflaton mass during the phase transition. This is discussed in Appendix A. However, nonadiabaticity can have other cosmological consequences as well. For example, abrupt turns in the trajectory of the inflaton in field space can not only likewise lead to particle production, but can also alter the primordial perturbation spectrum [46]. Within the context of our simple two-field model, this is not a concern: while the mixing generated by the phase transition does induce a turn in the inflaton trajectory, this occurs mostly prior to inflation, and the turn is sufficiently gradual that these effects may be safely neglected. However, these effects could play an important role in constraining 
other, more general models in which our slingshot mechanism is realized.

Along similar lines, the presence of additional heavy fields during inflation can affect the pattern of nonGaussianities in the CMB. Indeed, our slingshot mechanism relies on the mixing between the inflaton and other heavy fields in the scalar sector. Thus, signatures of this sort can potentially provide a way of testing our proposal. Indeed, imprints in the $\mathrm{CMB}$ resulting from the presence of such heavy fields have been studied extensively in the literature, both within the context of quasi-single-field inflation [47-49] and more generally via the analysis of other, "cosmological collider" signatures [50]. That said, the extent to which the pattern of non-Gaussianities is modified by these considerations is set primarily by the ratio $H / m_{\Phi}$, where $m_{\Phi}$ is the mass scale of the additional heavy field. Hence, while the effect of these fields on the resulting pattern of non-Gaussianities will be important for testing realizations of our mechanism with other kinds of dynamically-generated potentials-e.g., realizations which include heavy fields with masses closer to the Hubble scale - the size of this effect is typically small for the model considered in this paper since $H / \lambda_{1} \ll 1$.

Another natural question regarding our slingshot mechanism concerns whether it can be applied more than once. Although we have shown that an adequate number of $e$-folds of inflation can arise from a single slingshot, it would be interesting to investigate whether an additional, subsequent cosmological phase transition could induce a second slingshot, thereby propelling the lighter field in the scalar sector to even larger VEVs. Indeed, one might even imagine being able to obtain $N_{\text {inf }} \approx \mathcal{O}(50-60) e$-folds of inflation-or more-through a sequence of successive slingshots. At first glance, it might not seem that multiple slingshots could occur in succession, given that any radiation present in the universe prior to the onset of any inflationary epoch would be inflated away during that epoch and would thus no longer be present after inflation in order to trigger the next phase transition. This is not the case, however. As we have seen in Sec. III, the period of inflation precipitated by our slingshot mechanism typically begins only after both the corresponding phase transition and the kination-domination epoch which follows the slingshot have essentially concluded. This opens up the possibility that a second phase transition-and hence another slingshot-could occur after the end of the first phase transition but before inflation begins. Of course, this presupposes that a suitable set of initial conditions for the second slingshot arises after the first slingshot, and so forth. However, the state of the scalar sector at end of the kination-dominated epoch is one in which both fields are effectively at rest, in which a significant hierarchy exists between the masses of the two mass-eigenstates, and in which the lighter of these two states (which is sufficiently light that it effectively behaves as vacuum energy) is displaced from the minimum of the potential. These are precisely the initial conditions upon which our slingshot is built.

Unfortunately, a significant amount of energy density is dissipated from the scalar sector by Hubble friction during the kination-dominated epoch which follows each slingshot. For this reason, it may be challenging to obtain a significant overall enhancement in $\phi_{\lambda_{0}}^{(\mathrm{I})}$-and hence in $N_{\text {inf }}$-through successive slingshots. This issue is currently under study [51].

On a final note, we comment on the extent to which finetuning is required in order for our slingshot mechanism to yield an inflationary epoch of acceptable duration. As discussed in Sec. III, the parametric-resonance condition $\Delta_{G} \approx \Delta_{G}^{(n)}$ does not require a significant degree of finetuning. In fact, as we have seen in Fig. 8, a reasonable amount of fine-tuning would actually be required in order for $\phi_{\lambda_{0}}$ to avoid experiencing a substantial resonant enhancement upon its release from the slingshot. Similarly, we argued in Sec. III that we do not need to fine-tune our initial inflaton velocity $\dot{\phi}_{\lambda_{0}}\left(t_{\star}\right)$, provided that it has the correct sign. (Indeed, in other inflation scenarios this may no longer be true [52-59].) There is, however, one fine-tuning which is required in order for our slingshot mechanism to give rise to a suitable inflationary epoch. In order to ensure that we obtain an inflationary epoch of sufficiently long duration, our scenario requires a fairly large value of $\mathcal{Q}$, which in turn requires a significant degree of mixing between the two fields of our scalar sector, with $\bar{\beta} \ll 1$. However, this is tantamount to demanding that $\bar{\lambda}_{0} \ll H$. Although commonly a requirement in many models of inflation, such a small inflaton mass is unnatural unless protected by some symmetry (e.g., a global shift symmetry) and is generally difficult to control in the presence of Planck-suppressed operators. These issues constitute nothing other than the well-known $\eta$-problem. Of course, it has not been our goal in this paper to eliminate or ameliorate these fine-tuning problems; rather, our goal has been to provide a slingshot-based method of establishing viable initial conditions for inflation to occur. Our slingshot mechanism can nevertheless be viewed as reformulating these fine-tuning issues in terms of the mixing structure of a scalar sector, as established by a preinflationary phase transition. This could potentially provide an alternative route toward addressing these fine-tuning issues.

\section{ACKNOWLEDGMENTS}

We are happy to thank S. Watson for many helpful discussions. The research activities of K. R. D. are supported in part by the U.S. Department of Energy under Grant No. DE-FG02-13ER41976 (DE-SC0009913) and by the U.S. National Science Foundation through its employee IR/D program, while those of J. K. were supported by the Institute for Basic Science in Korea under project code 
IBS-R018-D1 and those of B. T. are supported in part by the National Science Foundation under Grant No. PHY1720430. Portions of this work were performed at the Aspen Center for Physics, which is supported in part by the National Science Foundation under Grant No. PHY1607611. The opinions and conclusions expressed herein are those of the authors, and do not represent any funding entities.

\section{APPENDIX: PARTICLE PRODUCTION IN THE SCALAR SECTOR}

In this paper, we have framed our discussion of the scalar-field dynamics associated with our slingshot mechanism largely in terms of the motion of the classical fields. However, certain quantum-mechanical effects can have an appreciable impact on the cosmology of our scalar sector and must also be taken into account. In particular, in scenarios in which the masses of the fields change abruptly, there can be significant production of particlelike excitations with comoving momenta $k$ and corresponding energies $\omega_{k}$ for which the adiabaticity condition

$$
\left|\frac{1}{\omega_{k}^{2}} \frac{d \omega_{k}}{d t}\right| \ll 1
$$

is violated [60-63]. Bounds on the energy density associated with such particlelike excitations of the fields in our two-scalar model ultimately lead to an upper bound on $N_{\text {inf }}$ for any given choice of model parameters. Indeed, any energy density pumped into particle production dilutes the energy density carried by the homogeneous scalar fields, thereby compromising the efficacy of our slingshot mechanism.

In this Appendix, we derive a rough estimate for the total energy density $\rho_{\lambda_{0}}^{(\mathrm{p})}$ of particlelike excitations generated during the phase transition in our model and assess the extent to which the presence of this energy density at the onset of inflation impacts our results. Since the heavier mass $\lambda_{1}^{2}$ typically evolves adiabatically before, during, and after the phase transition, we focus solely on the contribution associated with the lighter field $\phi_{\lambda_{0}}$, which plays the role of the inflaton.

In the mass eigenbasis, the evolution of $\phi_{\lambda_{0}}$ is governed by the equation of motion [17]

$$
\begin{aligned}
& \ddot{\phi}_{\lambda_{0}}+3 H \dot{\phi}_{\lambda_{0}}+\left(\lambda_{0}^{2}-\dot{\theta}^{2}\right) \phi_{\lambda_{0}} \\
& =-2 \dot{\theta} \dot{\phi}_{\lambda_{1}}-(\ddot{\theta}+3 H \dot{\theta}) \phi_{\lambda_{1}},
\end{aligned}
$$

where $\theta$ is the time-dependent mixing angle defined in Eq. (2.9). The mixing-dependent terms, which are induced by the rotation of the mass eigenbasis during the phase transition, are negligible within regions of parameter space which give rise to inflation. Dropping these terms and making the field redefinition $\varphi \equiv a^{3 / 2} \phi_{\lambda_{0}}$, we find that

$$
\ddot{\varphi}+\left[\lambda_{0}^{2}+\left(\frac{3}{2} H\right)^{2} w\right] \varphi=0,
$$

where $w$ is the equation-of-state parameter for the universe as a whole. At times soon before or after the phase transition, $\lambda_{0} \ll H$, and thus the second term inside the square brackets in Eq. (A3) provides the dominant contribution. However, during the phase transition the inflaton field becomes underdamped, with $3 H \lesssim 2 \lambda_{0}$, as a result of the pulse in the time development of $\lambda_{0}(t)$. During a brief interval around $t \sim t_{G}$, then, the first term in the square brackets in Eq. (A3) dominates. It is this interval during which the effects of nonadiabaticity are the most pronounced.

In order to estimate the amount of particle production due to these considerations, we must track the occupation numbers of all the momentum modes, treating the homogeneous zero mode of $\varphi$ as a classical, time-dependent background. We begin by recalling that the quantum inflaton field $\hat{\varphi}$ is represented in the Heisenberg picture as

$\hat{\varphi}(\mathbf{x}, t)=\int \frac{d^{3} k}{(2 \pi)^{3 / 2}}\left[\hat{a}_{\mathbf{k}} \varphi_{k}(t) e^{-i \mathbf{k} \cdot \mathbf{x}}+\hat{a}_{\mathbf{k}}^{\dagger} \varphi_{k}^{*}(t) e^{+i \mathbf{k} \cdot \mathbf{x}}\right]$,

where $\varphi_{k}(t)$ is the Fourier mode of $\varphi(t)$ with comoving momentum $k$, and where $\hat{a}_{\mathbf{k}}^{\dagger}$ and $\hat{a}_{\mathbf{k}}$ are the time-independent creation and annihilation operators for this mode. Each of the $\varphi_{k}(t)$ satisfies an equation of motion of the form

$$
\ddot{\varphi}_{k}+\omega_{k}^{2} \varphi_{k}=0
$$

where the corresponding frequency

$$
\omega_{k}^{2}=\frac{k^{2}}{a^{2}}+m_{\mathrm{eff}}^{2}
$$

inherits a time dependence both from the redshift of the physical momentum $k / a$ and from the evolution of the effective inflaton mass $m_{\text {eff }}$. This effective mass, which is given by

$$
m_{\mathrm{eff}}^{2} \equiv \lambda_{0}^{2}+\left(\frac{3}{2} H\right)^{2} w,
$$

evolves in time both as a result of the scalar-field dynamics associated with the phase transition and as a result of Hubble expansion. Moreover, while the first term in Eq. (A7) is completely specified by Eq. (2.13), the second depends on the state of the universe at times prior to the phase transition-and in particular on the equation-of-state parameter $w$ for the universe at such times. 


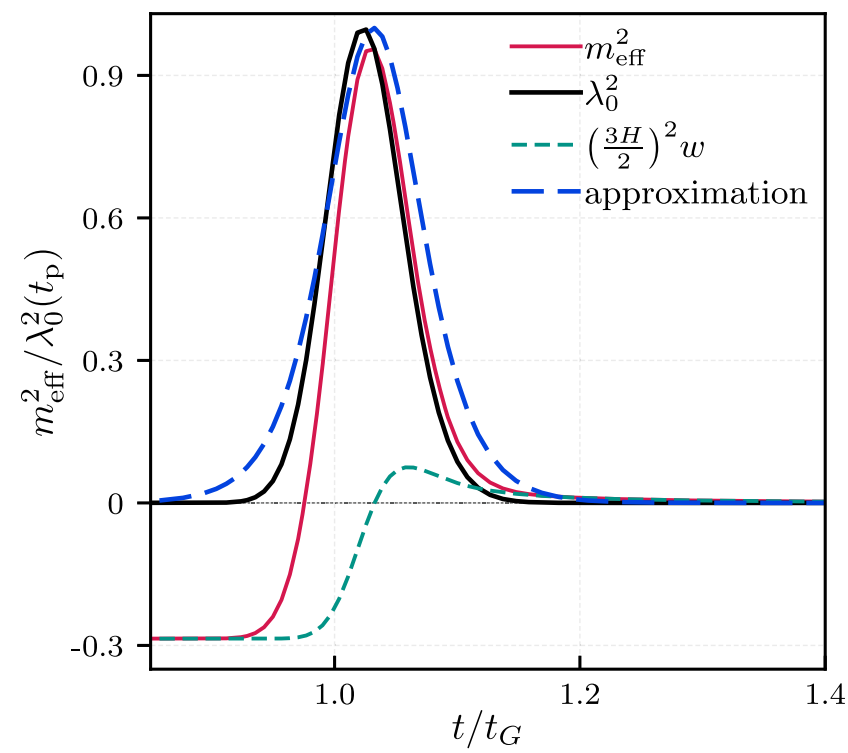

FIG. 13. The time development of the effective mass $m_{\text {eff }}^{2}$ of the inflaton in our two-scalar model in the vicinity of the pulse. The red curve shows the result obtained for an initial equationof-state parameter $w=-1$ for the universe immediately before the phase transition. The solid black and dashed green curves shown in the figure represent the corresponding individual contributions from the individual terms $\lambda_{0}^{2}$ and $(3 H / 2)^{2} w$ in Eq. (A7), respectively. The dashed blue curve represents the analytic approximation in Eq. (A8).

In Fig. 13, we show how the value of $m_{\text {eff }}^{2}$ evolves as a function of time during the phase transition, normalized to the value of $\lambda_{0}^{2}\left(t_{\mathrm{p}}\right)$. The red curve shows the result obtained for an initial equation-of-state parameter $w=-1$ for the universe immediately before the phase transition. The solid black and dashed green curves shown in the figure represent the contributions from the individual terms $\lambda_{0}^{2}$ and $(3 H / 2)^{2} w$ in Eq. (A7), respectively, as time evolves. We observe that in the vicinity of the pulse, where the change in the effective mass is most abrupt and the departure from adiabaticity is expected to be greatest, $\lambda_{0}^{2}$ represents the dominant contribution to $m_{\text {eff }}^{2}$.

In order to derive an approximate analytic expression for the energy density, we must determine the Bogoliubov coefficients which relate the asymptotic solutions to the equation of motion in Eq. (A5) for the $\varphi_{k}(t)$ at early times $t \ll t_{G}$, before the phase transition begins, to the corresponding asymptotic solutions at late times $t \gg t_{G}$, after it effectively concludes. Of course, the values for these coefficients depend on how the effective mass $m_{\text {eff }}^{2}$ evolves during the phase transition. For simplicity, and in order to make the parametric dependence of our results more transparent, we shall proceed by adopting a simple analytic approximation for $m_{\mathrm{eff}}^{2}$-an approximation which shall allow us to derive a corresponding analytic approximation for $\rho_{\lambda_{0}}^{(\mathrm{p})}$. In particular, we find that the function

$$
m_{\mathrm{eff}}^{2} \approx \lambda_{0}^{2}\left(t_{\mathrm{p}}\right) \operatorname{sech}^{2}\left(\frac{t-t_{\mathrm{p}}}{\frac{1}{2} \Delta_{G}}\right)
$$

provides a good approximation to both $\lambda_{0}^{2}$ and $m_{\mathrm{eff}}^{2}$ in the vicinity of the pulse. In fact, the blue dashed curve in Fig. 13 actually agrees well with the $\lambda_{0}^{2}$ curve throughout the time interval shown.

Unfortunately, the blue dashed curve does not agree with the $m_{\text {eff }}^{2}$ curve prior to the pulse. However, we expect that this deviation will not have a large impact on our results. The fact that $m_{\mathrm{eff}}^{2}<0$ in the asymptotic regime in which $t \ll t_{G}$ implies that the $\varphi_{k}(t)$ with $k \lesssim H a$ are tachyonic, with $\omega_{k}^{2}<0$. However, such modes, whose wavelengths exceed the Hubble radius at production, cannot properly be considered to be particlelike excitations [46]. These modes, which behave classically, therefore do not contribute to $\rho_{\lambda_{0}}^{(\mathrm{p})}$ (although they can affect the spectrum of density perturbations). It then follows that the spectrum of $\varphi_{k}(t)$ with higher momenta, which do contribute to $\rho_{\lambda_{0}}^{(\mathrm{p})}$, is approximately equivalent to the spectrum of modes which would be obtained had we replaced $m_{\text {eff }}^{2}$ with $\lambda_{0}^{2}$ in Eq. (A6). Given this, we shall approximate $m_{\text {eff }}^{2}$ as $\lambda_{0}^{2}$ in deriving our estimate for $\rho_{\lambda_{0}}^{(\mathrm{p})}$. We note, however, that the initial value of $w$ can have an impact on other inflationary observables.

In both the early-time and late-time asymptotic regimes, the inflaton potential evolves adiabatically and the $\varphi_{k}(t)$ are well approximated by $\varphi_{k}(t) \approx e^{-i \omega_{k} t} / \sqrt{\omega_{k}}$. It can therefore be shown that the problem of finding the Bogoliubov coefficients which relate these two sets of asymptotic solutions for $\varphi_{k}(t)$ is mathematically equivalent to the problem of determining the transmission coefficients for scattering off a potential $V(x) \propto-\operatorname{sech}^{2}(x)$ in nonrelativistic quantum mechanics [64]. Thus, by analogy, we find that the differential energy density $d \rho_{\lambda_{0}}^{(\mathrm{p})} / d k$ of inflaton field quanta per unit comoving momentum $k$ at the time the nonadiabatic evolution effectively ceases-which is roughly equivalent to the time at which $\phi_{\lambda_{0}}$ is released from the slingshot—-takes the form

$$
\frac{d \rho_{\lambda_{0}}^{(\mathrm{p})}}{d k} \approx \frac{4 \pi k^{2} \cos ^{2}\left[\frac{\pi}{2} \sqrt{1+\lambda_{0}^{2}\left(t_{\mathrm{p}}\right) \Delta_{G}^{2}}\right]}{(2 \pi)^{3} \sinh ^{2}\left(\frac{\pi}{2} \Delta_{G} k\right)} .
$$

We note that this differential energy density is largest for modes with momenta in the regime $k \lesssim \Delta_{G}^{-1}$. Integrating this energy density over $k$, we arrive at our estimate for the total energy density associated with particlelike excitations of the inflaton at the end of the phase transition. In particular, at this time we find that 


$$
\rho_{\lambda_{0}}^{(\mathrm{p})} \approx \frac{6 \zeta(3)}{\pi^{6} \Delta_{G}^{4}}\left[1+\cos \left(\pi \sqrt{1+\lambda_{0}^{2}\left(t_{\mathrm{p}}\right) \Delta_{G}^{2}}\right)\right]
$$

where $\zeta(x)$ denotes the Riemann zeta function.

In order for our slingshot mechanism to be successful, we must ensure that $\rho_{\lambda_{0}}^{(\mathrm{p})}$ is small in comparison with the energy density $\rho_{\lambda}$ associated with the classical background fields of the scalar sector. At the time at which the inflaton is released from the slingshot, $\rho_{\lambda_{0}}^{(\mathrm{p})}$ is given by Eq. (A10), while $\rho_{\lambda} \approx \frac{1}{2}\left[\lambda_{0}\left(t_{\mathrm{p}}\right) \mathcal{A}_{\phi}\right]^{2}$. Within our parameter-space region of interest, $\lambda_{0}^{2}\left(t_{\mathrm{p}}\right) \Delta_{G}^{2} \gtrsim 1$ and the ratio of these two energy densities at this time is therefore

$$
\frac{\rho_{\lambda_{0}}^{(\mathrm{p})}}{\rho_{\lambda}} \approx \frac{12 \zeta(3)}{\pi^{6} \lambda_{0}^{2}\left(t_{\mathrm{p}}\right) \mathcal{A}_{\phi}^{2}}\left(\frac{1}{\Delta_{G}^{4}}\right)
$$

During the subsequent kination-dominated epoch that precedes the onset of inflation, this ratio increases due to the fact that $\rho_{\lambda} \propto a^{-6}$ during this epoch, whereas $\rho_{\lambda_{0}}^{(\mathrm{p})}$, which is dominated by the contribution from relativistic momentum modes, scales like $\rho_{\lambda_{0}}^{(\mathrm{p})} \propto a^{-4}$. Thus, the ratio of these energy densities at the end of the kination-dominated epoch, when inflation begins, is

$$
\begin{aligned}
\left.\frac{\rho_{\lambda_{0}}^{(\mathrm{p})}}{\rho_{\lambda}}\right|_{\phi_{\lambda_{0}}^{\text {ini }}} & \approx \frac{12 \zeta(3)}{\pi^{6} \lambda_{0}^{2}\left(t_{\mathrm{p}}\right) \mathcal{A}_{\phi}^{2}}\left(\frac{e^{2 N_{\mathrm{kin}}}}{\Delta_{G}^{4}}\right) \\
& \approx \frac{12 \zeta(3) \mathcal{Q}^{2 / 3}}{\pi^{6} \lambda_{0}^{2}\left(t_{\mathrm{p}}\right) \Delta_{G}^{4}\left|\phi_{\lambda_{0}}^{(\mathrm{I})}\right|^{2 / 3}\left|\mathcal{A}_{\phi}\right|^{4 / 3}}
\end{aligned}
$$

where in going from the first to the second line we have used Eq. (4.2). Thus, we find that for a given choice of model parameters, the bound $\rho_{\lambda_{0}}^{(\mathrm{p})} \ll \rho_{\lambda}$ on the energy density of inflaton field quanta at the onset of inflation ultimately translates into a bound on $\mathcal{Q}$ of the form $\mathcal{Q} \ll \mathcal{Q}_{\max }$, where

$$
\mathcal{Q}_{\max } \equiv \frac{\pi^{9}}{24 \sqrt{3} \zeta^{3 / 2}(3)} \lambda_{0}^{3}\left(t_{\mathrm{p}}\right) \Delta_{G}^{6}\left|\phi_{\lambda_{0}}^{(\mathrm{I})}\right| \mathcal{A}_{\phi}{ }^{2}
$$

It is now straightforward to assess how this bound on $\mathcal{Q}$ constrains our slingshot model. For example, for our preferred parameter choices $\left|\mathcal{A}_{\phi}\right|=M_{p}, \bar{m}_{\text {sum }}^{2}=M^{2}$, and $\bar{\alpha}=0.9$ with $\Delta_{G}=\Delta_{G}^{(1)}$, we find from Eq. (A13) that $\mathcal{Q}_{\max } \approx 3.2 \times 10^{11}$. Comparing this upper bound on $\mathcal{Q}$ with the results shown in Fig. 10, we see that this bound indeed imposes a nontrivial constraint on our model. However, we also see that our slingshot mechanism can nevertheless yield a sufficient number of $e$-folds for cosmic inflation.

It is also important to note that while the bound on $\mathcal{Q}$ from particle production can be quite constraining, particularly for $\left|\mathcal{A}_{\phi}\right|<M_{p}$, there are several ways in which this bound can be relaxed in comparison to the benchmark quoted above. First, we note that this bound can be considerably weaker for higher-order parametric resonances than it is for the primary resonance. Indeed, since $\Delta_{G}^{(n)}$ increases with $n$-and in fact turns out to be roughly proportional to $n$-higher-order resonances require larger values of $\Delta_{G}$. Thus, given the explicit dependence of $\mathcal{Q}_{\max }$ on $\Delta_{G}$ in Eq. (A13), we expect larger values of $n$ to lead to significantly higher values of $\mathcal{Q}_{\max }$. While $\mathcal{Q}_{\max }$ also has an additional, implicit dependence on $\Delta_{G}$ through $\left|\phi_{\lambda_{0}}^{(\mathrm{I})}\right|$ which somewhat mitigates this effect, the suppression of $\left|\phi_{\lambda_{0}}^{(\mathrm{I})}\right|$ as $\Delta_{G}$ increases is quite gradual, as indicated in Fig. 8. Thus, the net impact of increasing $n$ is to raise $\mathcal{Q}_{\max }$ and thereby weaken the particle-production bound.

In this same connection, we also note from Fig. 8 that the parametric resonances in our slingshot model are quite broad. For example, the range of $\Delta_{G}$ values associated with the $n=1$ resonance spans several decades. Although the corresponding range of $N_{\text {inf }}$ is fairly narrow, the corresponding range of $\mathcal{Q}_{\max }$ is quite broad, extending over an order of magnitude or more. Thus, the bounds on $\mathcal{Q}$ obtained for a given resonance by taking $\Delta_{G}$ to be precisely equal to the corresponding $\Delta_{G}^{(n)}$ are likely to be overly conservative.
[1] A. A. Starobinsky, Phys. Lett. 91B, 99 (1980).

[2] A. H. Guth, Phys. Rev. D 23, 347 (1981).

[3] A. D. Linde, Phys. Lett. 108B, 389 (1982).

[4] A. Albrecht and P. J. Steinhardt, Phys. Rev. Lett. 48, 1220 (1982).

[5] K. A. Olive, Phys. Rep. 190, 307 (1990).

[6] D. Baumann, arXiv:0907.5424.

[7] W. H. Press, Phys. Scr. 21, 702 (1980).
[8] V. F. Mukhanov and G. V. Chibisov, JETP Lett. 33, 532 (1981).

[9] S. W. Hawking, Phys. Lett. 115B, 295 (1982).

[10] A. A. Starobinsky, Phys. Lett. 117B, 175 (1982).

[11] A. H. Guth and S. Y. Pi, Phys. Rev. Lett. 49, 1110 (1982).

[12] J. M. Bardeen, P. J. Steinhardt, and M. S. Turner, Phys. Rev. D 28, 679 (1983). 
[13] A. R. Liddle and S. M. Leach, Phys. Rev. D 68, 103503 (2003).

[14] A. D. Linde, Phys. Lett. 129B, 177 (1983).

[15] K. Freese, J. A. Frieman, and A. V. Olinto, Phys. Rev. Lett. 65, 3233 (1990).

[16] K. Freese and W. H. Kinney, Phys. Rev. D 70, 083512 (2004).

[17] K. R. Dienes, J. Kost, and B. Thomas, Phys. Rev. D 93, 043540 (2016).

[18] K. R. Dienes, J. Kost, and B. Thomas, Phys. Rev. D 95, 123539 (2017).

[19] D. Gorbunov and V. Rubakov, Introduction to the Theory of the Early Universe (World Scientific, Singapore, 2011), Vol. 2.

[20] Y. Akrami et al. (Planck Collaboration), arXiv:1807.06211.

[21] T. Moroi and T. Takahashi, Phys. Lett. B 522, 215 (2001); 539, 303(E) (2002).

[22] K. Enqvist and M. S. Sloth, Nucl. Phys. B626, 395 (2002).

[23] D. H. Lyth and D. Wands, Phys. Lett. B 524, 5 (2002).

[24] S. Mollerach, Phys. Rev. D 42, 313 (1990).

[25] A. D. Linde and V. F. Mukhanov, Phys. Rev. D 56, R535 (1997).

[26] M. Kawasaki, T. Kobayashi, and F. Takahashi, J. Cosmol. Astropart. Phys. 03 (2013) 016.

[27] T. Kobayashi, F. Takahashi, T. Takahashi, and M. Yamaguchi, J. Cosmol. Astropart. Phys. 10 (2013) 042.

[28] T. Futamase and K.-i. Maeda, Phys. Rev. D 39, 399 (1989).

[29] L. Boubekeur, E. Giusarma, O. Mena, and H. Ramírez, Phys. Rev. D 91, 103004 (2015).

[30] T. Tenkanen, J. Cosmol. Astropart. Phys. 12 (2017) 001.

[31] N. A. Chernikov and E. A. Tagirov, Ann. Inst. Henri Poincaré, A 9, 109 (1968).

[32] T. S. Bunch and P. C. W. Davies, Proc. R. Soc. A 360, 117 (1978).

[33] E. Ramirez and D. J. Schwarz, Phys. Rev. D 85, 103516 (2012).

[34] E. Ramirez, Phys. Rev. D 85, 103517 (2012).

[35] C. R. Contaldi, M. Peloso, L. Kofman, and A. D. Linde, J. Cosmol. Astropart. Phys. 07 (2003) 002.

[36] C. Destri, H. J. de Vega, and N. G. Sanchez, Phys. Rev. D 78, 023013 (2008).

[37] C. Destri, H. J. de Vega, and N. G. Sanchez, Phys. Rev. D 81, 063520 (2010).

[38] S. Downes and B. Dutta, Phys. Rev. D 87, 083518 (2013).

[39] E. Dudas, N. Kitazawa, and A. Sagnotti, Phys. Lett. B 694, 80 (2010).
[40] E. Dudas, N. Kitazawa, S. P. Patil, and A. Sagnotti, J. Cosmol. Astropart. Phys. 05 (2012) 012.

[41] N. Kitazawa and A. Sagnotti, J. Cosmol. Astropart. Phys. 04 (2014) 017.

[42] N. Kitazawa and A. Sagnotti, Mod. Phys. Lett. A 30, 1550137 (2015).

[43] M. Cicoli, S. Downes, and B. Dutta, J. Cosmol. Astropart. Phys. 12 (2013) 007.

[44] B. A. Powell and W. H. Kinney, Phys. Rev. D 76, 063512 (2007).

[45] I.-C. Wang and K.-W. Ng, Phys. Rev. D 77, 083501 (2008).

[46] M. Konieczka, R. H. Ribeiro, and K. Turzynski, J. Cosmol. Astropart. Phys. 07 (2014) 030.

[47] X. Chen and Y. Wang, J. Cosmol. Astropart. Phys. 04 (2010) 027.

[48] V. Assassi, D. Baumann, and D. Green, J. Cosmol. Astropart. Phys. 11 (2012) 047.

[49] T. Noumi, M. Yamaguchi, and D. Yokoyama, J. High Energy Phys. 06 (2013) 051.

[50] N. Arkani-Hamed and J. Maldacena, arXiv:1503.08043.

[51] K. R. Dienes, J. Kost, and B. Thomas (work in progress).

[52] R. Brandenberger, G. Geshnizjani, and S. Watson, Phys. Rev. D 67, 123510 (2003).

[53] A. Albrecht, R. H. Brandenberger, and R. Matzner, Phys. Rev. D 35, 429 (1987).

[54] D. S. Goldwirth, Phys. Lett. B 243, 41 (1990).

[55] D. S. Goldwirth and T. Piran, Phys. Rep. 214, 223 (1992).

[56] J. H. Kung and R. H. Brandenberger, Phys. Rev. D 42, 1008 (1990).

[57] J. H. Kung and R. H. Brandenberger, Phys. Rev. D 40, 2532 (1989).

[58] D. S. Goldwirth and T. Piran, Phys. Rev. Lett. 64, 2852 (1990).

[59] D. S. Goldwirth, Phys. Rev. D 43, 3204 (1991).

[60] N. D. Birrell and P.C. W. Davies, Quantum Fields in Curved Space, Cambridge Monographs on Mathematical Physics (Cambridge University Press, Cambridge, England, 1984).

[61] L. Kofman, A. D. Linde, and A. A. Starobinsky, Phys. Rev. Lett. 73, 3195 (1994).

[62] Y. Shtanov, J. H. Traschen, and R. H. Brandenberger, Phys. Rev. D 51, 5438 (1995).

[63] L. Kofman, A. D. Linde, and A. A. Starobinsky, Phys. Rev. D 56, 3258 (1997).

[64] M. A. Amin and D. Baumann, J. Cosmol. Astropart. Phys. 02 (2016) 045. 